\title{
Gene expression during zombie ant biting behavior reflects the complexity underlying fungal parasitic behavioral manipulation
}

Charissa de Bekker ${ }^{1,2 *}$, Robin A. Ohm³ ${ }^{3}$ Raquel G. Loreto ${ }^{2,4}$, Aswathy Sebastian ${ }^{5}$, Istvan Albert ${ }^{5,6}$, Martha Merrow ${ }^{1}$, Andreas Brachmann ${ }^{7}$ and David P. Hughes ${ }^{2^{*}}$

\begin{abstract}
Background: Adaptive manipulation of animal behavior by parasites functions to increase parasite transmission through changes in host behavior. These changes can range from slight alterations in existing behaviors of the host to the establishment of wholly novel behaviors. The biting behavior observed in Carpenter ants infected by the specialized fungus Ophiocordyceps unilateralis s.l. is an example of the latter. Though parasitic manipulation of host behavior is generally assumed to be due to the parasite's gene expression, few studies have set out to test this.

Results: We experimentally infected Carpenter ants to collect tissue from both parasite and host during the time period when manipulated biting behavior is experienced. Upon observation of synchronized biting, samples were collected and subjected to mixed RNA-Seq analysis. We also sequenced and annotated the O. unilateralis s.l. genome as a reference for the fungal sequencing reads.

Conclusions: Our mixed transcriptomics approach, together with a comparative genomics study, shows that the majority of the fungal genes that are up-regulated during manipulated biting behavior are unique to the O. unilateralis s.l. genome. This study furthermore reveals that the fungal parasite might be regulating immuneand neuronal stress responses in the host during manipulated biting, as well as impairing its chemosensory communication and causing apoptosis. Moreover, we found genes up-regulated during manipulation that putatively encode for proteins with reported effects on behavioral outputs, proteins involved in various neuropathologies and proteins involved in the biosynthesis of secondary metabolites such as alkaloids.
\end{abstract}

Keywords: Parasite-host interactions, Extended phenotype, Behavioral manipulation, Ophiocordyceps unilateralis, Camponotus castaneus, Genomics, Mixed transcriptomics

\section{Background}

Many parasites have evolved the ability to manipulate host behavior. This can range from alterations of existing behavioral traits to the expression of novel behaviors not present in the normal repertoire [1-3]. These changes in host behavior serve to promote completion of the parasite's life cycle and are generally assumed to be the result

\footnotetext{
*Correspondence: charissa.debekker@med.uni-muenchen.de; dhughes@psu.edu 'Institute of Medical Psychology, Faculty of Medicine,

Ludwig-Maximilians-University Munich, Goethestrasse 31, 80336 Munich, Germany

${ }^{2}$ Department of Entomology and Department of Biology, Center for Infectious Disease Dynamics, Pennsylvania State University, University Park, State College, Pennsylvania 16802 PA, USA

Full list of author information is available at the end of the article
}

of genes expressed by the parasite [3, 4]. Despite this, only a few studies have either demonstrated or implicated a genetic basis for manipulated behavior [5-12]. One example is insect infection by baculovirus where the virus induces an enhanced locomotory activity (ELA) in the caterpillar host prior to death $[5,11]$ combined with the migration of infected individuals towards elevated positions. At this elevated position, the host dies and liquefies, leading to virus particle transmission onto leaves that are then consumed by new caterpillar hosts [13-15]. ELA is a normal behavior in un-infected caterpillars, facilitating the search for suitable sites to undergo metamorphosis [16]. The baculovirus protein tyrosine phosphatase-encoding gene $p t p$, however, induces ELA upon infection $[5,11]$. A 
second mechanism involves expression of the baculovirus gene ecdysteroid uridine 5'-diphosphate (UDP)-glucosyltransferase (egt) that results in a disrupted climbing behavior [9]. Healthy caterpillars climb at night for feeding purposes and return down during the day to avoid predation. Upon infection, the retreat is disrupted due to egt expression. Despite the identification of these genes, the host pathways through which behavior is manipulated are still unknown. The attraction of infected individuals by light, however, suggests a role for host pathways involved in phototaxis and light perception [17].

The progress made in the baculovirus system does not necessarily provide us with answers that can be extrapolated to other systems. This is especially true in host-parasite systems where more complex manipulations are observed, which lead to manipulated hosts expressing wholly novel behaviors. One such example involves the fungal parasite Ophiocordyceps unilateralis sensu latu (s.l.) manipulating brains of Carpenter ants (genus Camponotus). In this system, a set of stereotyped behaviors is induced once the fungal colony growing inside the host reaches a sufficient size. Infection induced behaviors include leaving the nest at a different time of day compared to regular foraging, non-directed movements, convulsions and climbing up the vegetation [10]. Subsequently, the host bites into the vegetation at an elevated position and dies [18]. This manipulated biting is not part of the ant's normal behavior and assists the formation of the fruiting body and spore transmission post-mortem [18-20]. The place of biting is again stereotyped and seemingly adapted to the ecosystem in which the infection takes place: infected ants bite the main veins or margins of leaves in rainforests $[10,18,21,22]$ while they bite twigs in temperate forests [12]. Moreover, the transition from wandering to this death grip is synchronized to a certain time of day $[10,12]$.

Here, we describe the gene expression in the complex manipulator O. unilateralis s.l., a fungal isolate collected from the temperate forests of South Carolina [12], and its Camponotus ant host during the in vivo manipulated biting event. We performed a mixed transcriptomics study on the heads of experimentally infected individuals sampled during and after manipulated biting. We also sequenced and annotated the genome of O. unilateralis s.l. from North America. We found that during manipulated biting, the fungal parasite O. unilateralis s.l. up-regulates genes that putatively encode for proteins involved in oxidation-reduction processes and pathogenicity-related interactions, some of which may have medical or industrial applications. Moreover, we have identified genes that are involved in the expression of putative proteins that might affect host behavior. In the ant host, we found the differential expression of genes seemingly involved in apoptosis, immune and stress responses, as well as possible targets of behavioral manipulation.

\section{Results and discussion}

Apparent synchronization of manipulated biting behavior We used an O. unilateralis s.l. species from South Carolina and its natural host Camponotus castaneus to study behavioral manipulation of the host by the parasite. Ants were experimentally infected through injection and kept under $24 \mathrm{~h}$ light: dark (12 h: $12 \mathrm{~h}$ ) and temperature cycles together with sham-treated (injected with media without fungal material) and untreated individuals (see Methods). Only infected ants that died between 16 and 24 days post infection were observed in the characteristic manipulated biting position, as illustrated with pictures and videos in [12]. Manipulated biters were always found in this position during the first observational recording of the day at 09:00 $\mathrm{h}$ local time ( $3 \mathrm{~h}$ after lights on). The body and the legs would still be moving and twitching, an indication that the ant was alive. These ants would not react to any environmental stimuli (e.g., agitation, other ants). At 13:00 h, movements were reduced to occasional twitching of the legs. At 14:00 h no movement was detected, suggesting that the ant host had died. Similar observations were made in independent experiments with this parasite and host species (e.g., in [12]). The consistent observations of time of death imply that both, manipulated biting behavior and the subsequent death, are synchronized. Similar synchronized manipulation and death was observed in another species of ant-manipulating Ophiocordyceps from Thailand. However, in that system the infected ants displayed manipulated biting behavior around solar noon, followed by death $6 \mathrm{~h}$ after the biting event had taken place [10]. The shift of synchronized timing of biting towards the early morning/late night in our experiments could be an effect of the set-up (e.g., differences in temperature and light compared to nature), or due to species-specific timing of manipulated behavior. Future work to establish the timing of manipulation under field conditions in the temperate woods of South Carolina would be helpful.

Live ants displaying manipulated biting behavior were collected at 10:00 h. At 14:00 h, biting ants that showed no movement and thus appeared dead were harvested. This time point is referred to as "after manipulation". To decrease within-sample variation possibly introduced by collecting manipulated ants across different days, three individuals that were found manipulated on the same day were used for each sample type. Healthy control ants were also collected at 10:00 h and 14:00 h. As a baseline for $O$. unilateralis gene expression during and after manipulation, fungal cultures kept in insect cell culture media were harvested. 


\section{General genome features of 0 . unilateralis s.l. and C. floridanus}

As a reference for the C. castaneus RNA-Seq reads, the published genome of a related Camponotus species, Camponotus floridanus, was used [23]. For the present study, we repeated the functional annotations for the gene predictions using the same methodology as for O. unilateralis s.l.. This resulted in statistics similar to the ones previously reported: a total genome size of $234.88 \mathrm{Mb}$ encoding 17,061 genes of which 10,015 were predicted to encode proteins within 4,229 unique PFAM domains and of which $4.1 \%$ is putatively being secreted. The CEGMA completeness for this genome was $99.3 \%$.

The genome of the O. unilateralis s.l. from South Carolina [12], was sequenced to 120 -fold coverage. This genome served as a reference for the fungal RNA-Seq reads in our samples. Contig assembly resulted in a genome size of 26.05 megabases (Mb). Gene prediction yielded 7,831 putative genes. By using the Core Eukaryotic Genes Mapping Approach (CEGMA) core genes dataset [24, 25], the O. unilateralis s.l. genome was estimated to be $98.7 \%$ complete. PFAM domains were assigned to 5,556 (71 \%) of these genes, and there were 3,498 unique predicted PFAM domains [26]. The proportion of genes encoding putatively secreted proteins was $11.4 \%$. A Gene Ontology (GO) annotation was assigned to $49.9 \%$ of the genes and 1,800 (23 \%) received a Kyoto Encyclopedia of Genes and Genomes KEGG) annotation (Table 1). From these 1,800 genes, $45.9 \%$ were found to be involved in various forms of metabolism and $36.6 \%$ in genetic information processing such as transcription, translation and protein folding (Additional file 1).

The genome and gene predictions of O. unilateralis s.l. were compared to that of 16 published Ascomycota genomes. A phylogenetic reconstruction based on 168 single-copy genes that were conserved across the compared genomes is depicted in Fig. 1. The tree is congruent with a previously published phylogeny that contained a subset of the presently used organisms [27]. Fungi with various lifestyles both within and outside the class Sordariomycetes served as an outgroup to the order Hypocreales in our phylogenomic analysis. The order Hypocreales harbors a variety of insect parasites, (including O. unilateralis s.l.) distributed across several fungal families, as well as taxa that infect plants and other fungi (mycoparasites). We also assessed the conservation of the proteinencoding genes of O. unilateralis s.l. This revealed that $13 \%$ of all putative proteins within the genome are unique to O. unilateralis s.l., with $6 \%$ conserved only in fungi within the order Hypocreales, and $1 \%$ shared only with Ophiocordyceps sinensis. When we compared only those proteins that have a predicted secretion signal (SignalP annotation), $33 \%$ (=293 secreted proteins) were found to be unique to the O. unilateralis s.l. genome. Of these, 146
Table 1 Ophiocordyceps unilateralis s.l. genome assembly and annotation statistics

\begin{tabular}{ll}
\hline Property & Value \\
\hline Scaffolds in assembly & 7875 \\
Total assembly length (Mbp) & 26.05 \\
N50 (kb) & 22 \\
Largest scaffold (kb) & 145 \\
Assembly GC content (\%) & 54.76 \\
Assembly gaps (\%) & 0.73 \\
Repetitive content (\%) & 7.81 \\
Genes & 7831 \\
Gene length (median) & 1420 \\
Transcript length (median) & 1275 \\
Exon length (median) & 261 \\
CDS length (median) & 1272 \\
Protein length (median) & 424 \\
Spliced genes (total, \%) & 6038 (77.1\%) \\
Exons per gene (median) & 3 \\
Intron length (median) & 62 \\
Introns per spliced gene (median) & 3 \\
Gene density (genes/Mbp) & 300.57 \\
Proteins with internal stops (total, \%) & 0 \\
Unique PFAM domains & 3498 \\
Genes with PFAM (total, \%) & 5556 (70.95 \%) \\
Genes with GO (total, \%) & 3904 (49.85 \%) \\
Genes with signalP (total, \%) & $1493(19.07 \%)$ \\
Genes with TMHMM (total, \%) & 98.69 \\
CEGMA completeness (\%) & \\
\hline & \\
&
\end{tabular}

were predicted to be small secreted proteins (SSPs). This is $49 \%$ of all SSPs found in the O. unilateralis s.l. genome, which suggests that half of the putatively bioactive SSPs in this fungal parasite are unique to this species complex (Fig. 2). Both this set of unique SSPs and SSPs in general have a higher percentage of cysteine residues $(2.4 \%$ and $2.8 \%$, respectively), compared to the full protein set (1.4\%). This is frequently observed for SSPs in fungal genomes, and the cysteine residues likely play a role in disulphide bonds [28, 29].

We characterized the predicted secreted protein set further. An impressive number of 34 genes were found that contained one or more putative enterotoxin_a PFAM domains. These are bacterial-like toxins of which 9 are homologs of cholera toxins and the rest are homologs of heat-labile enterotoxin_a. These toxins are not commonly found in fungal genomes [30]. Of the 17 fungi analyzed here, 9 did not have any enterotoxin-encoding genes in their genomes. Only the insect-infecting fungi and plant pathogen $M$. oryzae contained genes putatively encoding 


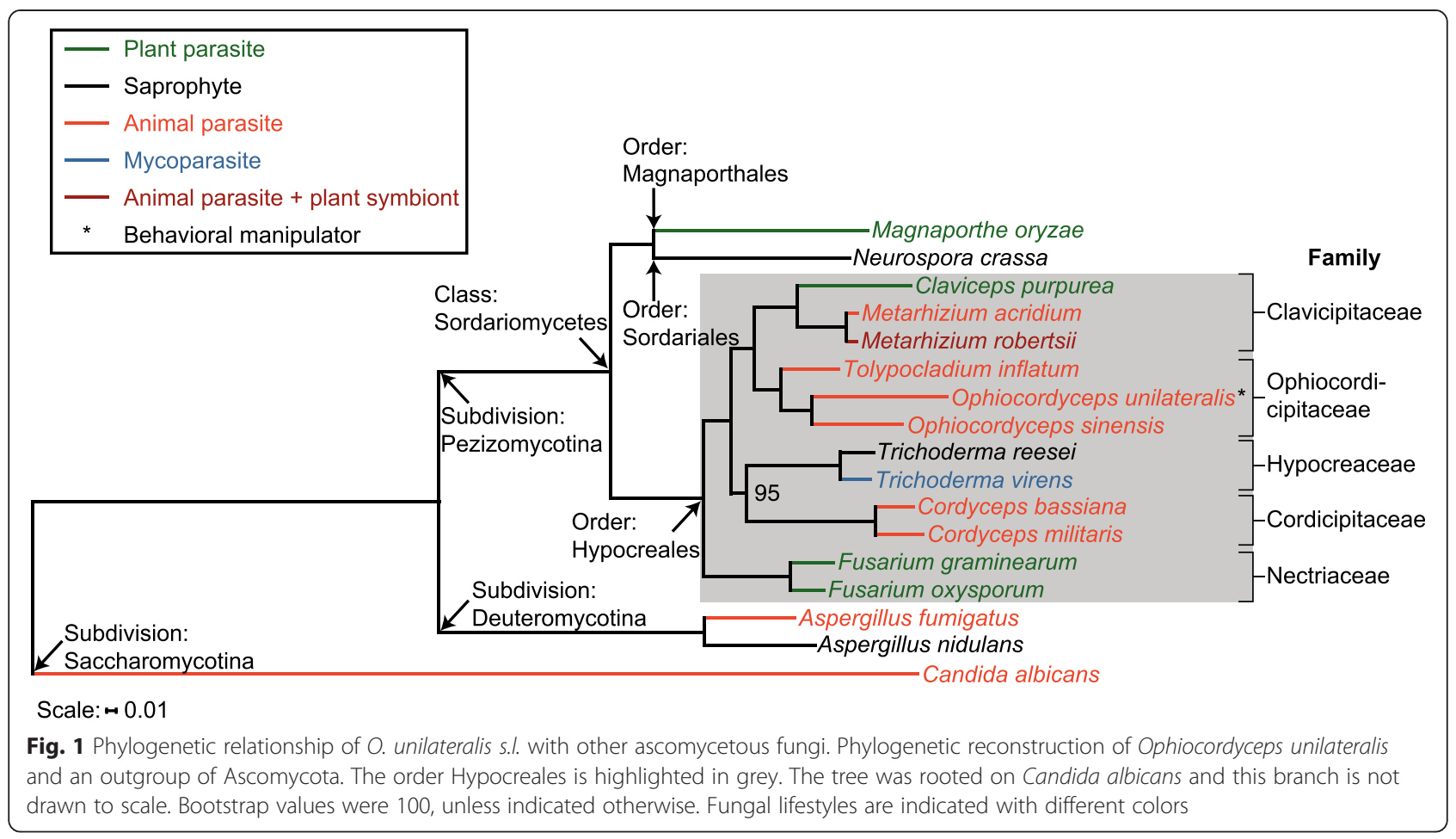

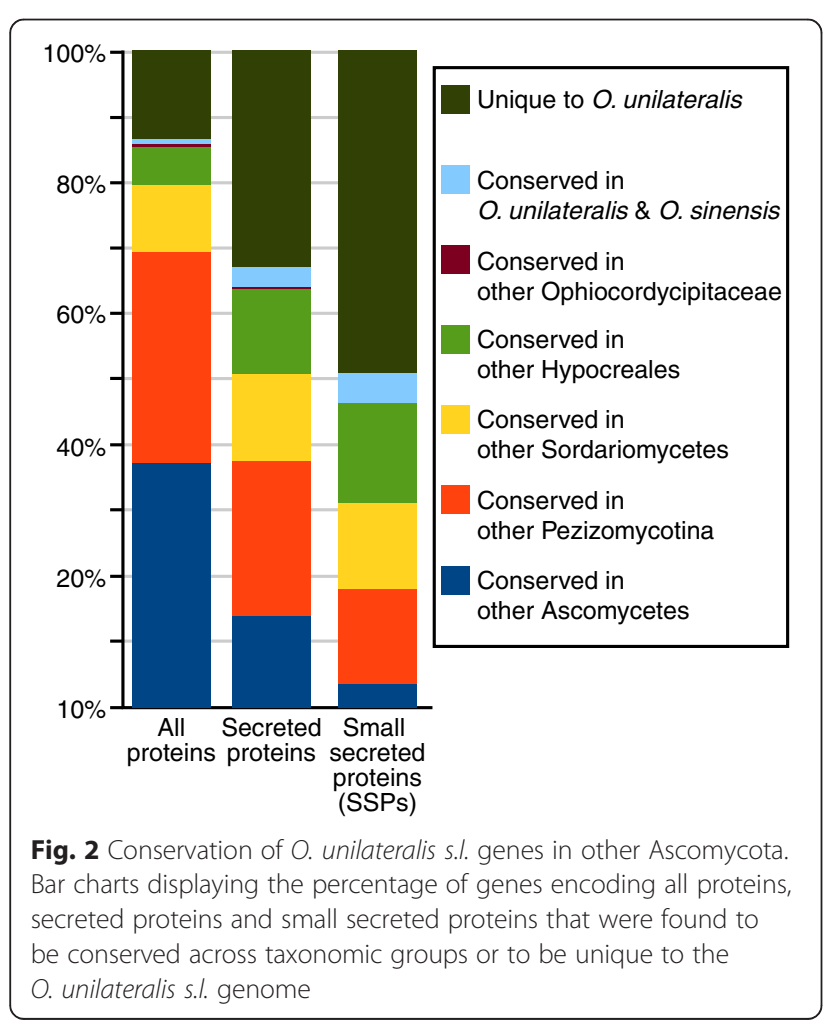

these bacterial-like toxins. The Tolypocladium inflatum genome was found to have 3 enterotoxin-encoding genes, Cordyceps militaris has 4, Magnaporthe oryzae has 6, Metarhizium acridium has 7, Ophiocordyceps sinensis has 8, Cordyceps bassiana has 14, and Metarhizium robertsii has 16. The O. unilateralis s.l. genome contained at least twice as many toxins relative to the other fungi within the comparison. A phylogenetic analysis showed that these enterotoxins are diversely distributed displaying resemblance with enterotoxins from other species. This indicates that the high number of enterotoxins in the genome of $O$. unilateralis are not a result of a duplication of a small number of enterotoxins, but rather a general expansion of phylogenetically diverse enterotoxin genes (Additional file 2). In weevils (a beetle), it has been shown that bacterial enterotoxins reduced the production of chemosignaling molecules by affecting the alcohols in these compounds [31, 32]. Communication in social insects generally involves chemical signals. As such, ants have an exocrine apparatus that is mainly used for this purpose [33]. The expansion of genes putatively encoding for secreted enterotoxins in the O. unilateralis s.l. genome might be an indication that impairing host communication through chemosignaling is one of the mechanisms employed by this manipulative fungus.

\section{General mixed transcriptome features of 0 . unilateralis s.l.} and $C$. castaneus

Gene expression profiles of O. unilateralis s.l. and $C$. castaneus during $(10: 00 \mathrm{~h})$ and after manipulated biting 
$(14: 00 \mathrm{~h})$ were generated through mixed transcriptomics on entire ant heads. We are therefore looking at the gene expression of interactions between the fungal parasite and the ant brain, as well as other tissues inside the head (e.g., muscles and fat body tissue). Gene expression studies on entire Drosophila heads however demonstrate that a good resolution of the processes in the brain can still be had this way [34]. Fungal cultures grown in insect cell culture media, and heads of healthy ants collected at the respective times of day for the mixed transcriptome samples, were used as controls. Sequencing yielded an average of 17.5 million paired reads per sample with an average mean quality score of 35 (see Additional file 3 for statistics). These reads were mapped to the O. unilateralis s.l. and C. floridanus reference genomes to obtain expression profiles, which were normalized to Fragments Per Kilobase of exon model per Million fragments mapped (FPKM) (see Methods). Of the O. unilateralis s.l. genes, 86.0, 86.5 and $85.0 \%$ were considered transcriptionally active with an expression level of at least 4 FPKM in the fungal control culture, during manipulation, and after manipulation, respectively. Of the C. floridanus homologs, 50.1 and $49.6 \%$ were considered transcriptionally active in C. castaneus controls compared to 50.0 and $49.8 \%$ in infected C. castaneus ant heads during and after manipulated biting behavior, respectively. Pairwise analysis was used to determine which genes were significantly $(\mathrm{Q}<0.05)$ and at least 2-fold up- or down-regulated between sample conditions (Additional file 4).

For the ant host, an average of $27.3 \%$ of the reads from the healthy control samples, $14.6 \%$ of the mixed "manipulated biting behavior" reads and $9.0 \%$ of the mixed "after manipulation" reads mapped to the $C$. floridanus genome (Additional file 3). The similarity/divergence of the C. castaneus and the C. floridanus genomes is unknown, which could be the reason for the lower percentage of reads mapping to the reference genome. Despite this low mapping score for ant reads, we did find 1,891 genes that were differentially expressed. This is considerably more compared to what has been found in previous work on gene expression of ant hosts challenged with pathogenic fungi (375 genes) [35]. For the fungal parasite, an average of $81.4 \%$ of the control sample reads, $46.9 \%$ of the mixed "manipulated biting behavior" reads and $61.1 \%$ of the mixed "after manipulation" reads mapped to the $O$. unilateralis s.l. genome. A relative comparison of the mixed transcriptome mapping scores to those of the single transcriptomes suggests that during manipulated biting behavior only about half of the tissue inside an infected ant head was made up by the ant host $(27.3 \%$ of the control sample reads vs. $14.6 \%$ of the "after manipulation" reads mapping to the C. floridanus genome). The other half consisted of fungal cells $(81.4 \%$ of the control sample reads vs. $46.9 \%$ of the "after manipulation" reads mapping to the O. unilateralis s.l. genome). After manipulation had taken place, the fungal colony had grown to make up about $75 \%$ of the biological material inside an infected ant's head (Additional file 5). An alternative explanation is that the expression levels considerably decreased in the dying ant, which causes the relative expression of the fungus to increase.

\section{Enrichment of functional annotations in O. unilateralis s.l. differential gene expression}

To obtain insight into the biological processes that are different between samples, we performed an enrichment analysis of functional annotations among differentially expressed genes. We determined which functional annotation terms were significantly overrepresented among various subsets of differential expression (Additional files 6 and 7 ) by performing the Fisher Exact test (corrected $\mathrm{P}<0.05)$. Within the 1,417 genes that were up-regulated in O. unilateralis s.l. during the manipulated biting $(10: 00 \mathrm{~h})$ compared to fungal control cultures, those involved in DNA binding, DNA replication and DNA repair, and oxidation-reduction processes were overrepresented (Additional file 6: Table S1). Genes involved in DNA replication and DNA repair were also overrepresented in the 1,050 up-regulated fungal genes after manipulated biting had taken place (14:00 h) compared to control cultures (Additional file 6: Table S3).

Genes involved in the metabolism of sugars were generally down-regulated during manipulation (Additional file 6: Table S2). However, a direct comparison between gene expression in O. unilateralis during (10:00 h) and after manipulation (14:00 h) indicated an overrepresented up-regulation of carbohydrate processing genes after manipulation had taken place (Additional file 6: Table S5). Even when the data set was narrowed down to only those genes that were first down-regulated during manipulation before being up-regulated again afterwards, carbohydrate metabolic processes were still overrepresented (Additional file 6: Table S8). This suggests that the ants collected at 14:00 $\mathrm{h}$ indeed represent the situation after manipulation in which the host has presumably been killed and the fungus has transitioned to actively consuming the host (i.e., saprophytic growth). Following death, rapid growth occurs as the fungus switches from growing within the host to growing externally, forming the stalk needed for reproduction [18]. Coinciding with this, genes with a pathogenesis GO annotation term were overrepresented in the down-regulated gene set after manipulation had taken place (Additional file 6: Table S4). All pathogenesis-related genes in this subset were annotated as protein exotoxins with either a functional enterotoxin_a domain (all but one) or a Pertussis S1 subunit domain (Additional file 7, sheet 4). As 
discussed above, such exotoxins are normally secreted by bacteria but have also been reported to be present in fungal genomes such as C. bassiana [30].

Our transcriptomics data thus indicate a shift from parasitic to saprophytic activity. This is supported by previous metabolomics analyses, which demonstrated that fungal parasites display vastly different secretomes during growth on live versus dead ant tissues [36]. Related to this shift we found that after manipulation, O. unilateralis s.l. had downregulated an overrepresented amount of genes involved in oxidation-reduction processes, such as cytochrome P450s (CYPs) (Additional file 6: Table S6). One of the many roles CYPs play involves the biosynthesis of secondary metabolites related to pathogenesis [37]. This result still holds when the subset of genes is reduced to only those that were first significantly up-regulated during manipulation (about $60 \%$ of this subset, Additional file 6: Table S7). Genes with a secondary metabolism annotation term were also downregulated in overrepresented manner after manipulation, when compared to expression during biting. Among them were genes putatively involved in the metabolism of neurotoxic compounds such as alkaloids and indole diterpene alkaloids (penitrems), and genes involved in the production of fungal bioactive compounds such as nonribosomal peptide synthetases (NRPSs) and polyketide synthases (PKSs) (Additional file 7, sheet 7).

When examining the subsets for differential expression we found an overrepresentation of genes predicted to encode secreted proteins in almost all of them. When comparing gene expression during manipulation with that of the fungal control culture, 13.5 and $14.7 \%$ from the total of the up- and down-regulated genes, respectively, were annotated to have a secretion signal. This is, respectively, 21.4 and $19.0 \%$ of all genes predicted to encode secreted proteins (Additional file 6: Tables S1 and S2). The fungus $O$. unilateralis s.l. thus likely secretes a different array of compounds inside the ant at the time of manipulated biting, compared to when it is grown in insect cell culture media. Similarly, about $20 \%$ of all secreted protein encoding genes was down-regulated after manipulation had taken place, both when compared to the fungal control culture and to gene expression during manipulation (Additional file 6: Tables S4 and S6). This suggests that, again, after manipulation, the fungal parasite dynamically changes its secretion profile. Our findings are in agreement with previous work using a metabolomics approach to determine the secretomes of fungal entomopathogens (including O. unilateralis s.l.) in insect cell culture media with and without ant tissues $[12,36]$. About one third of the up- and down-regulated genes encoding secreted proteins were annotated as SSPs (Additional file 7). SSPs are often highly speciesspecific, lacking similarity to known proteins, making functional predictions difficult. Several SSPs that have been experimentally characterized are involved in parasitic relationships of fungi with their hosts, affecting cellular processes inside the host [38, 39]. This suggests that $O$. unilateralis s.l. not only has a specific secretion profile during behavioral manipulation, it also tailors its array of small secreted effector proteins.

Since secreted proteins were generally overrepresented, we also investigated their conservation across the entomopathogenic (insect-infecting) fungi within the order Hypocreales. From all the genes that were annotated to have a secretion signal, about $42 \%$ were conserved among the fungi with an insect parasitic lifestyle. A little over half of the secreted proteins was thus to be found only in the O. unilateralis s.l. genome. Of this $58 \%$, about $12 \%$ was up-regulated solely during manipulated biting behavior, while this was only $6.7 \%$ for the conserved putative secretion proteins. In fact, the majority $(82 \%)$ of the genes found up-regulated during manipulated biting behavior and down-regulated again after, were not conserved in other Hypocrealean entomopathogens (Fig. 3). This suggests that O. unilateralis s.l. might be using a rather unique set of genes to establish

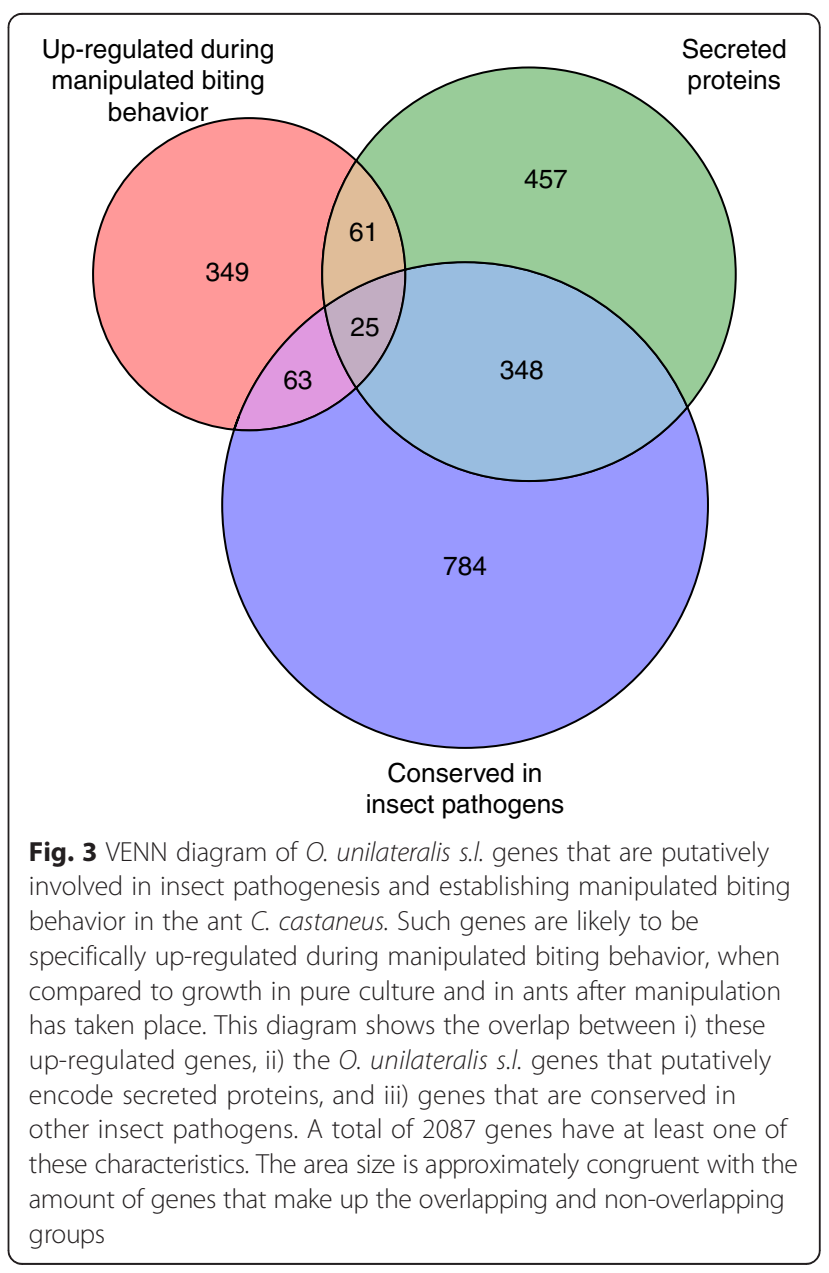


manipulated biting behavior, compared to other nonmanipulating insect-infecting fungi.

\section{Up-regulation of alkaloid biosynthesis during manipulated biting behavior}

In general, behavior-manipulating parasites are thought to change host behavior by means of secreting neuromodulatory agents near both the central and peripheral nervous systems of their hosts [40]. Secondary metabolites, such as polyketides, nonribosomal peptides and alkaloids, have been suggested to play a role in this [41]. We hypothesized that genes encoding for secreted candidate neuromodulators, and proteins involved in the biosynthesis of neuromodulating secondary metabolites would be up-regulated during manipulated behavior, and likely down-regulated again afterwards (gene subset in Additional file 8, sheet 2). We indeed found PKSs and NRPSs with this expression pattern, as well as proteins involved in the production of various alkaloids (summarized in Table 2). For example, two tryptophan dimethylallyltransferases within the O. unilateralis s.l. genome were highly expressed (426 and 938 FPKM) during manipulated biting behavior while transcription was nonexistent in the fungal baseline culture. Their expression also significantly dropped again after manipulation had taken place and the ant host had died (Additional file 8, sheet 2). Tryptophan dimethyltransferases catalyze the first rate-limiting step of ergot alkaloid biosynthesis of which tryptophan is the biochemical precursor [42, 43]. Indeed, a gene putatively encoding for tryptophan synthase followed a similar expression pattern suggesting accumulation of the precursor during manipulation as well. Moreover, 2 putative indoleamine 2,3-dioxygenases, which degrade tryptophan, were significantly downregulated at this time (Additional file 7, sheet 3). Ergot alkaloids are well-known to affect animal behavior. They are a class of indole alkaloids, which resemble neurotransmitters such as serotinin, noradrenaline and dopamine and can therefore act on the central and peripheral nervous systems as agonists of various receptors [44]. Claviceps purpurea, a related fungus infecting rye grasses, produces these compounds [42]. The ergot in these infected grasses has been known to cause serotonergic overstimulation of the Central Nervous System in humans and livestock that consumed infected rye, which can lead to e.g., muscle spasms and hallucinations [45]. This suggests that the putative production of ergot-like alkaloids by $O$. unilateralis s.l., through the up-regulation of genes involved in their metabolism, could be affecting the brains of Carpenter ants in similar fashion.

Tryptophan synthesis in turn involves the precursors anthranilate and indole-3-glycerol-phosphate [46]. The latter can also function as an indole donor to synthesize indole diterpene alkaloids, such as the fungal compound paxilline $[47,48]$. Several genes putatively encoding enzyme homologs of the pax pathway (i.e., the biosynthesis pathway of fungal indole diterpenes) were found to be highly expressed in O. unilateralis s.l. during the manipulated biting event, again followed by a significant downregulation after manipulation had taken place (Additional file 7, sheet 7). A putative geranyldiphosphate transferase (PaxC homolog) was found, which catalyzes the first step in this pathway. In addition, a flavin-dependent monooxygenase (PaxM homolog) and an integral membrane protein (PaxB homolog) were found, which are required for the following oxidative cyclization steps resulting in a wide variety of indole diterpenes and their precursors paspaline and emindole DA $[49,50]$. These metabolites, also called penitrems, can have a variety of biological activities, including the modulation of insect and animal ion channels and insect feeding deterrence [51, 52]. Furthermore, they can inhibit big potassium (BK) channels, which regulate processes such as passive smooth muscle contraction and neuron excitation causing tremors (i.e. unintentional rhythmic muscle movement) [53]. A continuous rhythmic moving of the legs of manipulated twig-biting individuals was observed (in this study as well as [10] and [12], additional videos), which indicates that the putative penitrem production by $O$. unialteralis s.l. might have a tremorgenic effect in ants as well.

Since tryptophan can also function as the precursor for many other classes of alkaloids (KEGG Ontology Pathway 00400) we searched for more differentially expressed genes involved in tryptophan metabolism. Among the genes involved in tryptophan metabolism that were significantly up-regulated both during and after manipulation we found a histidinol-phosphate aminotransferase (Additional file 7, sheet 2 and 4). KEGG Orthology indicated that this enzyme could be involved in the biosynthesis of the tropane alkaloid scopolamine. This compound is an antagonist of the muscarinic acetylcholine receptor, which binds the neurotransmitter acetylcholine (reviewed in [54]). Similarly, the putative production of tropane alkaloids by O. unilateralis s.l. could have an antagonist effect on neurotransmitter receptors within the membranes of ant brain neurons.

\section{Differentially expressed 0 . unilateralis s.l. genes with secretome annotations}

In almost all subsets for O. unilateralis s.l. genes that were differentially expressed during and after manipulated biting behavior, those that were annotated to have a secretion signal were overrepresented. As previously stressed, this indicates that this parasitic fungus dynamically changes a significant part of its secretome from the time of manipulation to host death a few hours after manipulation (Additional file 9). During manipulated biting behavior, 191 genes encoding secreted proteins were up-regulated, of which 61 were SSPs (Additional file 7, sheet 2). From 
Table 2 Candidate O. unilateralis s.I. neuromodulators

\begin{tabular}{|c|c|c|c|}
\hline Protein ID & Functional annotation & $\begin{array}{l}\text { Fold up during } \\
\text { manipulation }\end{array}$ & $\begin{array}{l}\text { Fold down death } \\
\text { after manipulation }\end{array}$ \\
\hline \multicolumn{4}{|c|}{ Secondary metabolism } \\
\hline Ophio1_1/g767.t1 & $\begin{array}{l}\text { Putative tryptophan dimethyltransferase involved in metabolism of ergot alkaloids, } \\
\text { which resemble indole neurotransmitters and are therefore agonists of various } \\
\text { neuroreceptors }\end{array}$ & Infinite & 40.46 \\
\hline Ophio1_1/g3491.t1 & $\begin{array}{l}\text { Geranylgeranyldiphosphate transferase, PaxC homolog involved in metabolism of indole } \\
\text { diterpenes (penitrems) causing tremors through big potassium channel inhibition }\end{array}$ & 18688.55 & 25.57 \\
\hline Ophio1_1/g3493.t1 & $\begin{array}{l}\text { Geranylgeranyl pyrophosphate synthase involved in metabolism of terpenoids and } \\
\text { polyketides }\end{array}$ & 14705.25 & 18.60 \\
\hline Ophio1_1/g3831.t1 & Putative PKS & 4313.78 & 4.85 \\
\hline Ophio1_1/g3492.t1 & $\begin{array}{l}\text { Integral membrane protein, PaxB homolog involved in metabolism of indole diterpenes } \\
\text { (penitrems) causing tremors through big potassium channel inhibition }\end{array}$ & 3445.96 & 16.75 \\
\hline Ophio1_1|g3487.t1 & $\begin{array}{l}\text { Flavin-dependent monooxygenase, PaxM homolog involved in metabolism of indole } \\
\text { diterpenes (penitrems) causing tremors through big potassium channel inhibition }\end{array}$ & 3015.11 & 39.72 \\
\hline Ophio1_1|g3485.t1 & $\begin{array}{l}\text { Putative tryptophan dimethyltransferase involved in metabolism of ergot alkaloids, which } \\
\text { resemble indole neurotransmitters and are therefore agonists of various neuroreceptors }\end{array}$ & 2293.83 & 28.51 \\
\hline Ophio1_1/g768.t1 & NRPS-like protein & 374.06 & 19.74 \\
\hline Ophio1_1/g444.t1 & Homolog of P450 involved in fusarin C metabolism cluster & 147.52 & 12.25 \\
\hline Ophio1_1/g4784.t1 & Putative NRPS & 6.14 & 8.84 \\
\hline Ophio1_1/g3877.t1 & Putative NRPS & 6.03 & 3.77 \\
\hline Ophio1_1|g5818.t1 & $\begin{array}{l}\text { Putative histidinol-phosphate aminotransferase involved in metabolism of tropane } \\
\text { alkaloid scopolamine, which has an antagonist effect on neuroreceptors }\end{array}$ & 2.91 & 1.17 \\
\hline Ophio1_1|g1532.t1 & Acyl-hydrogenase involved in metabolism of terpenoids and polyketides & 2.52 & 2.28 \\
\hline \multicolumn{4}{|l|}{ Secreted proteins } \\
\hline Ophio1_1|g67605.t1 & $\begin{array}{l}\text { SSP, putative lipocalin, ApoD homolog, lipophilic protein binding capacity, involved in } \\
\text { brain neuropathologies }\end{array}$ & Infinite & 17.39 \\
\hline Ophio1_1/g209.t1 & SSP, no functional annotation & Infinite & 124.98 \\
\hline Ophio1_1/g2647.t1 & SSP, no functional annotation, unique to O. unilateralis s.I. & Infinite & 74.44 \\
\hline Ophio1_1/g2296.t1 & $\begin{array}{l}\text { SignalP, putative bacterial-like enterotoxin involved in pathogenesis and possibly } \\
\text { affecting chemosensory compounds }\end{array}$ & 3263.32 & 199.43 \\
\hline Ophio1_1/g4844.t1 & $\begin{array}{l}\text { SignalP, putative sphingomyelin phosphodiesterase, ASM, SMPD1 homolog involved in } \\
\text { sphingolipid metabolism and therefore cell regulation }\end{array}$ & 1506.00 & 7.06 \\
\hline Ophio1_1/g5103.t1 & SSP, contains ankyrin-repeat, unique to $O$. unilateralis s.l. & 316.29 & 3.24 \\
\hline Ophio1_1|g5079.t1 & SSP, no functional annotation & 118.50 & 3.23 \\
\hline Ophio1_1/g3495.t1 & SSP, no functional annotation, unique to O. unilateralis s.I. & 113.50 & 2.91 \\
\hline Ophio1_1/g364.t1 & $\begin{array}{l}\text { SignalP, putative protein-tyrosine-phosphatase, involved in ELA behavior induction in } \\
\text { Lepidoptera by baculoviruses }\end{array}$ & 111.28 & 5.39 \\
\hline Ophio1_1/g4465.t1 & $\begin{array}{l}\text { SSP, putative kynurenine formamidase involved in tryptophan metabolism towards } \\
\text { quinolinic acid and kynurenic acid }\end{array}$ & 102.17 & 6.26 \\
\hline Ophio1_1/g734.t1 & SSP, no functional annotation, unique to O. unilateralis s.I. & 85.48 & 19.28 \\
\hline Ophio1_1/g6878.t1 & SSP, no functional annotation & 59.57 & 3.82 \\
\hline Ophio1_1/g1605.t1 & SSP, no functional annotation & 51.39 & 11.53 \\
\hline Ophio1_1/g3218.t1 & SSP, no functional annotation & 29.14 & 2.92 \\
\hline Ophio1_1|g3121.t1 & SSP, no functional annotation & 20.03 & 2.84 \\
\hline Ophio1_1/g6661.t1 & SSP, no functional annotation, unique to O. unilateralis s.l. & 17.31 & 7.61 \\
\hline Ophio1_1|g1154.t1 & SSP, no functional annotation, unique to 0 . unilateralis s.I. & 17.02 & 2.53 \\
\hline Ophio1_1/g2359.t1 & SSP, no functional annotation, unique to O. unilateralis s.I. & 12.19 & 3.56 \\
\hline Ophio1_1/g749.t1 & SignalP, P450, CYP5A ortholog, putative thromboxane-A synthase regulating & 10.39 & 6.36 \\
\hline
\end{tabular}


Table 2 Candidate O. unilateralis s.I. neuromodulators (Continued)

\begin{tabular}{llll}
\hline Ophio1_1lg103.t1 & $\begin{array}{l}\text { SignalP, tyrosinase involved in dopamine metabolism, melanin production and quinone } \\
\text { production }\end{array}$ & 8.70 & 21.89 \\
Ophio1_1lg7417.t1 & SSP, no functional annotation & 8.45 & 2.43 \\
Ophio1_1lg3227.t1 & SSP, no functional annotation, unique to O. unilateralis s.l. & 6.90 & 3.36 \\
Ophio1_1lg5581.t1 & SSP, no functional annotation, unique to O. unilateralis s.l. & 4.64 & 17.27 \\
Ophio1_1lg25.t1 & SSP, no functional annotation, unique to O. unilateralis s.l. & 3.72 & 2.97 \\
Ophio1_1lg999.t1 & $\begin{array}{l}\text { SignalP, tyrosinase involved in dopamine metabolism, melanin production and quinone } \\
\text { production }\end{array}$ & 2.05 & 6.12 \\
\hline
\end{tabular}

these SSPs, about half was transcribed $>10$-fold more during manipulation compared to the baseline expression and 28 were significantly down-regulated again after the event had taken place (Additional file 8, sheet 2). Of these, 16 did not receive a functional annotation. Our genome comparison analysis showed that 9 of these SSP genes were unique to the genome of $O$. unilateralis as well as one gene that contained an ankyrin repeat. Among those that were functionally annotated were 2 putative hydrophobic surface binding proteins (HsbA), 1 hydrophobin domaincontaining protein, 1 cysteine-rich secretory antigen, 5 pathogenesis-related (CAP) protein, and a toxic ricin $\mathrm{B}$ lectin. Another SSP was GO annotated to have hydrolase activity and to be involved in a metabolic process. This gene was $>100$-fold up-regulated during manipulation and significantly down-regulated 6-fold again after. A homology search against the NCBI database suggested that this SSP is a putative kynurenine formamidase (Additional file 8 , sheet 2). This hydrolase is involved in the kynurenine pathway of tryptophan metabolism [55], which produces nicotinamide adenine dinucleotide (NAD). NAD is involved in redox reactions by being an oxidizing agent. A putative up-regulation of its production through a high expression of kynurenine formamidase could therefore be involved in the overall up-regulation of oxidation-reduction processes seen during manipulation (see section below). Quinolinic acid and kynurenic acid are also formed through the kynurenine pathway. Their production could therefore also be increased through the high expression of this putative kynurenine formamidase. The formation of an excessive amount of quinolinic acid through the kynurenine pathway and failing to maintain physiological concentrations of kynurenic acid have been reported to have neuropathological effects [56-58]. Moreover, disturbance of the balance between the various pathways of tryptophan metabolism, such as the suggestive kynurenin pathway upregulation in our data, could possibly result in serotonin depletion. In ants, serotonin depletion diminishes the likelihood that workers respond to the foraging trails, and when they do so, they walk shorter distances [59]. Indeed, field observations reported that ants infected by O. unilateralis s.l. do not walk on the trails and stay within the nest vicinity
[60]. Taken together, the up-regulation during manipulation of this secreted hydrolase (Table 2 and Fig. 4), of which its homolog is involved in pathways that can cause neurological disorders, suggests another candidate possibly involved in establishing manipulated behavior in $O$. unilateralis s.l-infected ants.

Another candidate involved in manipulation is an SSP annotated to have a Lipocalin-2 domain (Table 2 and Fig. 4). A homology search suggested that this gene encodes for a D-like apolipoprotein (ApoD), which is a close ortholog to invertebrate Lazarillo-related proteins [61]. This SSP was not expressed in the fungal control culture, highly expressed during manipulation of ant behavior and 17-fold down-regulated again after manipulation (Additional file 8, sheet 2). Lipocalins are extracellular proteins found in bacteria, plants, vertebrates and invertebrates [62], including the order Hymenoptera in which the ants reside [61]. Homologs have also been reported from two other fungal genomes [63]. Our genome comparison analysis found homologs in M. oryzae (confirming [63]), C. bassiana and C. militaris. Lipocalins can be functionally diverse with roles ranging from the storage and transport of chemically sensitive or insoluble metabolic products [64], to participating in olfaction and chemical communication $[65,66]$, and regulation of (neuronal) stress and immune responses [67]. This makes lipocalin proteins interesting candidates in drug discovery. O. unilateralis could thus potentially secrete lipocalins as a way to regulate stress responses during manipulation of behavior, deliver secondary metabolites to the brain tissue, or alter chemical communication. Future functional assays are however needed to determine this.

Among the rest of the secreted proteins that were upregulated during manipulated biting behavior and downregulated again after manipulation, were 9 putative proteases: 6 serine proteases ( 1 tripeptidyl-peptidase and 5 subtilisins), 2 aspartyl proteases, and 1 metallocarboxypeptidase (Additional file 8 , sheet 2). Pathogenic fungi commonly secrete these protease families. Though the function of fungal secreted proteases and their importance in infections may vary across species, these enzymes are 


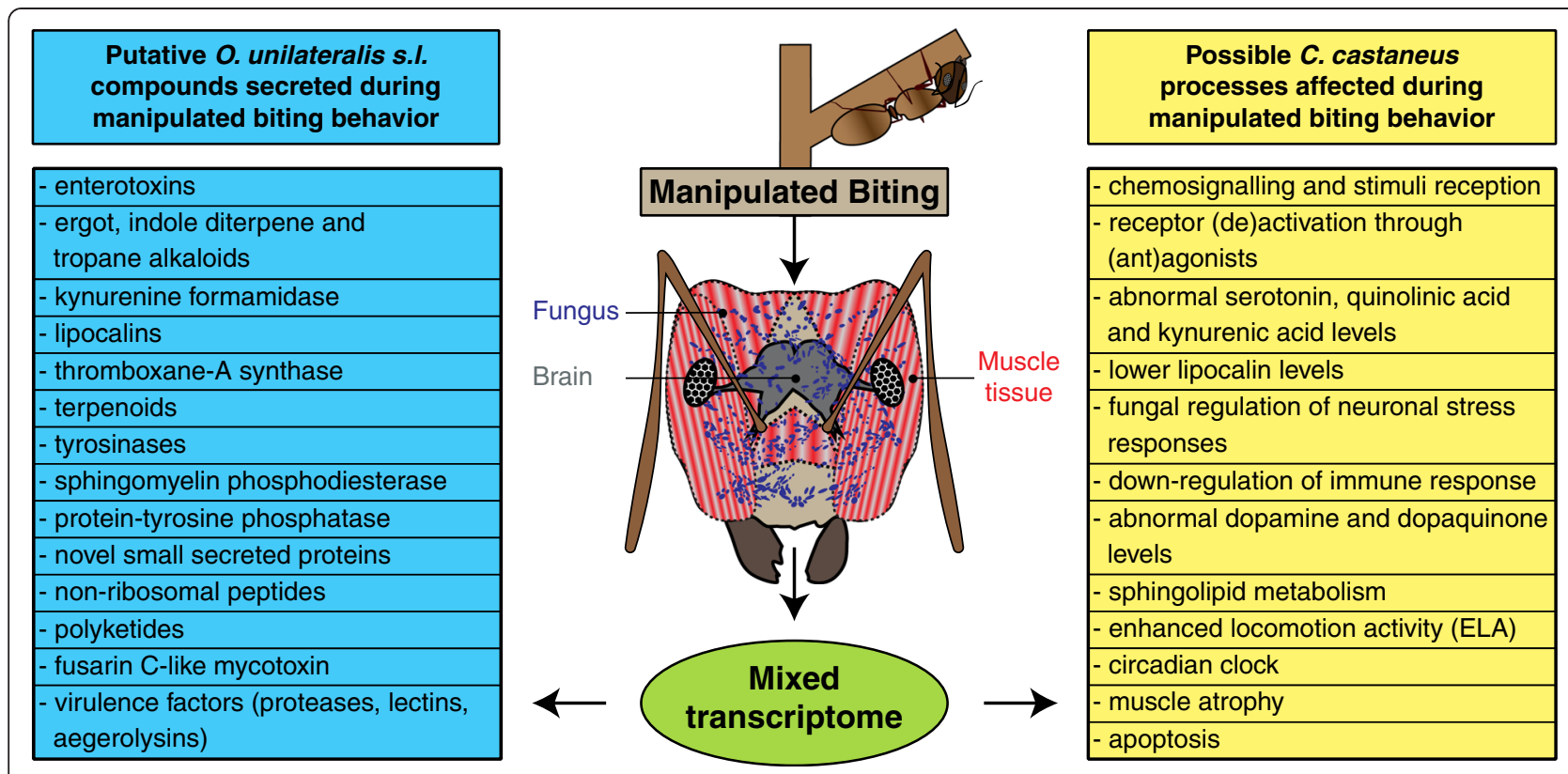

Fig. 4 Infographic summarizing the putatively secreted O. unilateralis s.l. compounds and possible C. castaneus processes found in this study that are seemingly involved in manipulation

largely considered important virulence factors [68]. Another putatively secreted protein that was up-regulated $>1,500$-fold during manipulation (Additional file 8, sheet 2) was annotated as a sphingomyelin phosphodiesterase. In fact, KEGG annotation indicated that this gene is a homolog of acid sphingomyelinase (ASM) SMPD1, an important enzyme in sphingolipid metabolism. Biological membranes consist of sphingolipids, together with cholesterol and phospholipids. As such, sphingolipids are involved in all types of cell regulation through cell recognition and cell signaling [69]. Neuronal cells are especially affected by defects in sphingolipid metabolism with defects in ASM specifically resulting in the neurological disorder NiemannPick syndrome [70]. This suggests that O. unilateralis s.l. could change neuron cell regulation through the secretion of a putative ASM in large amounts during manipulation, which is in line with the reported enrichment of the metabolite sphingosine in ex vivo O. unilateralis s.l. - C. castaneus ant brain interactions [12]. Moreover, the muscular atrophy accompanying manipulation and vegetation biting has been hypothesized to be the result of altered nerve motor neuron innervation [10]. Sphingomyelinase has, however, also been found to be an important factor for Bacillus cereus pathogenicity through the induction of host epithelial cell death in concert with the secretion of enterotoxins [71]. The upregulation of secreted ASM could therefore also be complementing enterotoxin secretion, which suggests that this gene may be a virulence factor contributing to pathogenesis. The two effects manipulation and cell death may indeed be related as the biting behavior, which is the hallmark of O. unilateralis s.l. pathogenicity, does involve extensive atrophy [10].

Another secreted enzyme whose gene is being upregulated during manipulation (>110-fold) and significantly down-regulated after $(>5$-fold), is a putative protein-tyrosine phosphatase (PTP) (Additional file 8, sheet 2). In fact, 2 more genes putatively encoding for this enzyme are significantly higher expressed inside the ant's head compared to growth in culture (Additional file 7, sheet 2). For two baculoviruses, Bombyx mori nucleopolyhedrovirus and Autographa californica nucleopolyhedrovirus, the gene encoding for PTP was found to manipulate behavior of their respective insect hosts (B. mori and Spodoptera exigua). In these cases PTP induced enhanced locomotion activity (ELA) [5, 11]. ELA induced by baculoviruses occurs late in the infection during which infected caterpillars move to the upper plant foliage where they die. Similarly, we find Ophiocordyceps-infected ants biting at elevated positions. This suggests that $O$. unilateralis s.l. up-regulates putative secreted PTPs possibly causing enhanced locomotion behavior in ant hosts to ensure manipulated biting will take place outside of the nest and at an elevated position (Table 2 and Fig. 4).

\section{Differentially expressed $O$. unilateralis s.l. genes involved in oxidation-reduction processes}

Among the genes that were significantly up-regulated during manipulated biting behavior, and down-regulated after manipulation had taken place, those involved in oxidationreduction processes were significantly enriched (Additional file 6: Tables S1, S6 and S7). This annotation is rather 
general since many essential cellular processes require the oxidation and/or reduction of molecules involved. Interference in the balance between oxidants and reductants leads to oxidative- or reductive-stress conditions, which can result in inflammation, carcinogenesis, degenerative - and other diseases (reviewed in [72]). Moreover, parasite redox biology is unfolding to be an important aspect of parasitehost interactions. These recent studies have shown that host immune responses include oxidative attacks and the production of antioxidant enzymes by the parasite is considered vital for virulence, disease progression and outcome [73, 74]. During manipulation, 134 genes that were GO annotated to encode for proteins with an oxidationreduction function were up-regulated (Additional file 7, sheet 2). After manipulated biting, 65 were significantly down-regulated again (Additional file 8, sheet 2). From this subgroup of differentially expressed genes, 15 were annotated to encode the cytochrome $\mathrm{P} 450$ proteins (CYPs) discussed above. These monooxygenases contribute to processes such as the biosynthesis of secondary metabolites, the assimilation of carbon sources, carcinogenesis, detoxification and degradation of xenobiotics [37, 75]. Of these CYPs, two were involved in secondary metabolism clusters through catalyzing the modification of intermediate products as "decorating" enzymes (Additional file 8, sheet 2). Both were actively transcribed during manipulated biting while transcription was basically absent during baseline expression and after manipulation had taken place. Another differentially expressed secretion signal-containing CYP was predicted to be involved in arachidonic acid metabolism working as a thromboxane-A synthase (CYP5A ortho$\log$ ). Arachidonic acid is released from cell membranes by inflammatory stimuli and oxidized into compounds such as thromboxanes. These have been implicated as critical mediators in inflammatory diseases in vertebrates [76]. However, little or nothing is known about thromboxanes in insects at present. This suggests again that the fungal parasite might be regulating inflammation levels inside the ant's head at the time of manipulated biting (Table 2 and Fig. 4). One CYP was predicted to be involved in phenylalanine metabolism and xenobiotics biodegradation working as a phenylacetate 2-hydroxylase. A fifth CYP with a predicted metabolism function was indicated as a CYP3A ortho$\log$ involved in steroid hormone biosynthesis, linoleic acid metabolism and retinol metabolism. This CYP was also KEGG annotated to have carcinogenic activities (Additional file 8, sheet 2). CYP3A members have largely been studied because of their dramatic accumulation induced by a diverse array of xenobiotics [77], which suggests that its up-regulation in O. unilateralis s.l. is due to the interactions with a live host and the stressors involved in such a process.

In addition to these CYPs, four other oxidation-reduction related secondary metabolite cluster genes were found to be up-regulated only during manipulated biting. One of these was annotated to encode for an NRPS-like enzyme and therefore suggested to be involved in the production of bioactive non-ribosomal peptides. The other three were "decorating" dehydrogenases predicted to be involved in i) steroid biosynthetic processes, ii) metabolism of bioactive terpenoids and polyketides that are widely used as therapeutic drugs and pharmaceutical agents [78], and iii) the biosynthesis of streptomycin, a compound that can have both antibiotic and toxic effects (Additional file 8, sheet 2). This KEGG ortholog for streptomycin biosynthesis is also homologous to a CYP within the Fusarin $\mathrm{C}$ cluster. Fusarin $C$ is a mycotoxin with carcinogenic effects produced by several plant pathogenic Fusarium species [79] as well as insect pathogen Metarhizium [80]. This suggests that this up-regulated oxidoreductase might be involved in the biosynthesis of a fungal secondary metabolite with similar pathogenic effects for the host.

Of the secreted oxidoreductase activity containing proteins that were found to be up-regulated during manipulated biting behavior, four were annotated to function as tyrosinases (Additional file 7, sheet 2). Of those, two were significantly down-regulated again after manipulation had taken place (Additional file 8, sheet 2). Tyrosinases are type- 3 copper proteins involved in melanin synthesis from tyrosine. They catalyze the hydroxylation of monophenols into diphenols and facilitate the oxidation of those into reactive quinones. As such, tyrosinases are especially responsible for the first steps resulting in the formation of dopaquinone [81] but have also been shown to play a role in other parts of dopamine synthesis and metabolism in the brain [82]. In fungi, tyrosinase is mainly associated with melanin production (i.e., browning and pigmentation). The functions of these melanins range from mechanisms of defense and resistance to environmental stresses [83] to virulence mechanisms [84]. Moreover, in insects, melanin plays a major role in the innate immune system by damaging and encasing invading microbes [85]. However, when dissecting O. unilateralis s.l. infected ants, no browning of the mycelium is observed [18] while browning of both the mycelium and the medium is seen in Ophiocordyceps cultures grown in culture [12]. The expression of tyrosinase was 2-8.7-fold higher during manipulated biting behavior when compared to expression during growth in culture. This suggests that tyrosinase might be up-regulated not merely to produce melanin. Research indeed shows that the quinones generated from dopamine and DOPA by tyrosinase are cytotoxic both in and beside dopaminergic neurons through the interaction with a variety of bioactive molecules [86]. Moreover, quinone formation has been related to pathogenesis in Parkinson's disease [82], mitochondrial dysfunction in the brain [87], inflammation leading to degeneration of dopamine neurons as 
seen in methamphetamine neurotoxicity $[88,89]$ and proteasome impairment [90]. The specific up-regulation of fungal tyrosinases during the behavioral manipulation of the ant host might therefore exert more than a mere virulence or environmental stress response function (Table 2 and Fig. 4).

\section{Differentially expressed pathogenicity-related O. unilateralis s.l. genes}

Since this mixed transcriptomics study aims to reveal the gene expression at a particular time point during a parasite-host interaction, an up-regulation of pathogenicityrelated genes in the parasite is to be expected. We indeed found putative pathogenesis-related genes from different classes, some of which have already been discussed above. During manipulated biting behavior we also found 2 ricintype lectins, 1 fucose specific lectin and 1 lectin-like flocculation protein to be significantly higher expressed compared to both expression in the baseline culture and heads of ants after manipulation had taken place (Additional file 8, sheet 2). Lectins bind carbohydrates and mediate recognition in parasite-host interactions. They are highly sought after because of their great therapeutic and biotechnological potential. The roles of many fungal lectins remain elusive, however functions such as host recognition and yeast flocculation have been proposed [91]. Furthermore, a ricintype lectin from Rhizoctonia solani was found to induce significant toxicity in insect pests [92]. Another protein with interesting biotechnological potential that was found to be up-regulated during manipulation was a putative aegerolysin. Fungal aegerolysins are said to have antitumoral, anti-proliferative, and anti-bacterial activities as well as being involved in the early stages of fructification [93]. A homology search for this gene revealed that this $O$. unilateralis s.l. aegerolysin shares $63 \%$ similarity with Asp-hemolysin of A. fumigatus, which is a lethal and cardiotoxic component of this pathogenic fungus [94]. This suggests that $O$. unilateralis s.l. up-regulates an array of possible virulence factors when growing inside its ant host. These are essential to understand the parasite-host interactions taking place, as well as of potential biotechnological interest for the fields of medical drug discovery and biological pest control.

The genome comparison (discussed above) showed that O. unilateralis s.l. has far more genes encoding secreted putative bacterial-like enterotoxins compared to other fungi studied so far. Of the 34 putative enterotoxins in the O. unilateralis s.l. genome, 12 were not transcriptionally active under the conditions used in this study. Of the transcriptionally active ones, only two were constitutively expressed across the conditions tested leaving a total of 21 differentially expressed enterotoxins. During manipulated biting behavior, six enterotoxins were up-regulated compared to expression during growth in culture. Two of these were significantly down-regulated again after manipulation had taken place. Nine were down-regulated during manipulated biting behavior, of which three were significantly up-regulated again afterwards. This highly dynamic expression of the majority of enterotoxins indicates that their expression is tailored towards the environmental conditions that $O$. unilateralis s.l. encounters and different life cycle stages.

\section{Pathology-related changes in C. castaneus: immune reaction and apoptosis}

So far we have discussed changes in the gene expression of the fungal parasite during and after manipulated biting behavior (summarized in Fig. 4). The mixed transcriptomics approach, allows us to also investigate gene expression of the ant host at these time points (also summarized in Fig. 4). Because this is a host-parasite system it might be expected that immune-related gene expression in the host is altered because of parasitism. Previous studies on gene expression in ants have shown an up-regulation of immune-related genes upon infection by bacteria [95] and the generalist fungus Metarhizium brunneum $[35,96]$. These studies sampled the ants in the early stages of the infection, 24 and $48 \mathrm{~h}$ after the entry of the pathogen respectively. In our study we sampled more than two weeks after the entry of the parasite as this amount of time is needed to establish manipulation [12]. We found that many of these immune-related genes were down-regulated in ant heads at time of manipulated biting compared to heads of healthy ants. This included genes involved in pathogen recognition (e.g. peptidoglycan recognition protein), signaling (e.g. cytochrome p450 $6 \mathrm{k} 1$ ) and immune response effectors, such as prophenoloxidase and antimicrobial peptides (AMPs) (defensin 1, defensin 2 and a homolog of dynamin) (Additional file 10, sheets 4 and 5). Other genes important in insect immune and stress responses, such as the homologs of malvolio and punch [97], were constitutively expressed across the controls and infected samples. Moreover, our enrichment analysis revealed that among the 391 down-regulated genes in the head of the live host during manipulated biting, those related to oxidoreductase activity were overrepresented (Additional file 7: Table S6). This suggests that O. unilateralis s.l. might be able to suppress host immune responses in the head during the event of manipulated biting behavior. Similarly, stress-related genes were also differentially expressed in infected ants. Again, the Ophiocordyceps infection resulted in down-regulation of most common stress-related genes in insects, such as the homolog of the insect lipocalin lazarillo [98]. However, at the same time, a secreted fungal lipocalin was up-regulated (discussed above) implying that the parasite might be regulating the host's neuronal stress response. 
Although parasites suppressing the expression of ants' immune responses have not been previously documented, it has been shown for other hymenopterans (ants belong to the order Hymenoptera). The ectoparasitic mite Varroa destructor suppresses the innate immune system of honeybees (Apis melifera) by reducing the expression of AMPs and immunity-related enzymes [99]. The bumblebee Bombus terrestris has its immune response affected by the trypanosome gut parasite Crithidia bombi through the down-regulation of AMPs [100]. Furthermore, other trypanosome parasites that cause vector borne diseases, such as Trypanosoma brucei (sleeping sickness) and Leishimania (leishmaniasis), also repress immune reaction related expression in their insect vectors (tsetse and sandflies, respectively) $[101,102]$. All three of these parasites are co-evolved specialists unlike the generalist pathogens used in $[35,96]$. We suggest that O. unilateralis s.l., like other specialist parasites, might suppress the immune response in heads of the ants it infects.

At the moment of manipulation, the fungal cells occupy the head of the ant host, causing severe atrophy and a reduction in organelles of muscle cells, but leaving the brain preserved [10]. However, $48 \mathrm{~h}$ post-mortem, brain tissue was virtually indistinguishable from the fungal cells [18]. In line with this previous evidence of tissue breakdown, we found the differential expression of a number of apoptosis-related genes during the manipulation, compared to the healthy controls. Overall, proapoptotic genes were found to be up-regulated during the manipulated biting event (Additional file 10, sheet 4). These included proteins annotated as feminization-1 (Fem1b) homolog b-like and specificity protein-3 transcription factor (Sp3). Fem1b plays a role in sex determination in Caenorhabditis elegans regulating cell elimination through apoptosis [103, 104]. In humans, the up-regulation of its homologue (FEM-1) induces apoptosis via the caspase pathway $[105,106]$. The zinc finger transcription factor $\mathrm{Sp} 3$ is important for the regulation of genes implicated in cell cycles and programed cell death [107]. Its overexpression results in apoptosis of cancer cells [107-109] and cortical neuron cells in culture [110]. Sp3 was also found to be accumulated in the postmortem brain tissue of patients with Alzheimer disease, and has been suggested to play a role in the neurodegenerative progression characteristic of Alzheimer, which in humans results in cognitive loss [110]. We suggest that preserving the brain during colonization of the head may be necessary for $O$. unilateralis s.l. to successfully manipulate behavior. However, after the ant host is successfully attached to a substrate (the time point at which we collected the manipulated ants for RNA-Seq analysis), the fungus appears to either induce neural apoptosis or release hydrolytic enzymes that cause host tissue degradation to be able to complete its development.

\section{Possible ant host targets of behavioral manipulation}

Prior to manipulated biting, infected ant hosts display a stereotypical walking pattern, followed by constant climbing and falling from the vegetation due to whole body convulsions [10]. This suggests neuronal activity is affected. In line with this, in the head of the ant sampled at 10:00 $\mathrm{h}$ during the manipulated biting, we found the differential expression of putative biogenic amine precursors and receptors. Biogenic amines are known to influence behavior of both vertebrates $[111,112]$ and invertebrates [113-117], including ants [118-120]. We for instance found the up-regulation of a putative beta-3R-like octopamine receptor gene and a tyrosine 3 -monooxygenase gene (Additional file 10, sheet 4). Tyrosine 3-monooxygenase is the rate-limiting enzyme for the biosynthesis of dopamine [121]. Although dopamine is a precursor for the melanin pathway in the immune response of insects (ants included: [35, 95]), it was demonstrated in Drosophila melanogaster that dopamine also functions as a neurotransmitter in the fly nervous system. Furthermore, the expression of tyrosine 3-monooxygenase appears to oscillate in a circadian manner in D. melanogaster, regulating motor neuron activity and neuromuscular functions through the biosynthesis of dopamine [122]. This results in the circadian regulation of locomotion behavior [121]. The up-regulation of this enzyme could therefore be involved in establishing the aberrant walking behavior that is observed in O. unilateralis s.l. infected ants [10]. Moreover, increases in the dopamine levels in Formica polyctena ants (by direct injection) were found to stimulate the opening of mandibles and biting [120]; behaviors that were both observed in manipulated ants moments before the biting event. This could indicate that the up-regulation of tyrosine 3-monooxygenase plays a role in the behavioral changes observed in $O$. unilateralis s.l.-infected ants.

In addition to walking aberrantly, manipulated ants do not follow trails like their healthy nest mates [10, 60]. One possible explanation is that the manipulated ants receive limited stimuli from the environment. We indeed found genes putatively encoding for receptors and binding proteins involved in odorant and gustatory perception (e.g. OR-1 and ORCO) were down-regulated during manipulated biting behavior relative to gene expression in healthy ant heads (Additional file 10, sheet 5). Making the host latch on in the optimal microenvironment for fungal growth and spore dispersion is crucial to the life cycle of this parasite $[18,60]$. The erratic walking has been observed in nature to last at least three hours [10]. Suppressing the sensorial perception could therefore be a strategy to keep the ant engaged in this process. However, mosquitoes infected with the generalist fungal pathogen $C$. bassiana, which does not manipulate host behavior as a way to achieve transmission, were found to 
be less responsive to feeding stimuli, as well [123]. The lack of response to stimuli could therefore also be a general pathology-related host reaction or an ancient characteristic of entomopathogenic fungi in general.

Another characteristic feature of the manipulation of C. castaneus behavior by $O$. unilateralis s.l. is the switch to diurnality. Uninfected $C$. castaneus are primarily nocturnal ([124] and our extensive field observations). Moreover, the manipulated biting behavior and subsequent death appears to be synchronized to a certain time of day, both in the laboratory and in nature (this study and [10], respectively). This has led to the hypothesis that the fungus might affect the circadian clock of the host [12]. The circadian clock is a temporal program that anticipates the highly predictable daily changes of the environment. The molecular mechanism of the biological clock is conserved across different groups of organisms [125], exemplified by positive and negative transcriptional feedback loops on one hand, and the redox state of peroxiredoxin on the other hand [126]. In insects, two of these clock genes are period and cycle. Fire ant (Solenopsis invicta) period and cycle oscillate in anti-phase in their transcript levels [127]. In manipulated ants $(10: 00 \mathrm{~h})$, period and cycle homologs were expressed with a 2.3 and 2.1-fold increase, respectively, compared to uninfected controls sampled at 10:00 $\mathrm{h}$ (Additional file 10, sheet 5). This may affect the expression of clock-controlled genes regulating certain behaviors such as the above-mentioned oscillating tyrosine 3monooxygenase in Drosophila [121, 122]. The circadian clock in some organisms regulates cell cycle control, proliferation and apoptosis $[128,129]$. For instance, the over- and under-expression of homologous clock genes PER1 [130] and BMAL1 [131] in human cells correlates with the rapid proliferation of aggressive tumor cells and to tumor cell control through apoptosis [132, 133], respectively. This is consistent with our apoptosis-related findings discussed above and previously reported morphological data [10].

O. unilateralis s.l.-infected ants display increased and constant activity before the manipulated biting takes place [10]. This is suggestive of the increased locomotor activity in caterpillars infected with baculovirus [5]. In caterpillars, this behavior is related to the expression of $p t p$. We also found a significant up-regulation of PTPencoding genes during manipulation, both in the ant (Additional file 10, sheet 4), as well as in the fungal transcriptome (Additional files 7 and 11, sheet 2). The observed enhanced locomotion in behaviorally manipulated ants could be a result of this up-regulated $p t p$ expression. Ultimately, the manipulation of ant host behavior results in biting the vegetation at an elevated position, which fixes the ant to a substrate and is crucial for fungal development and transmission [18, 60]. The deep penetration of the mandibles into the plant tissue and the atrophy of the mandibular muscles cause the "lock-jaw", ensuring the lasting attachment of the ant even once it has been killed by the fungus. The muscle atrophy is characterized by muscle fiber detachment from the head capsule, with broken z-lines (anchors for muscle contraction) and less dense sarcoplasmic reticula and mitochondria [10]. In our transcriptomics study, we found that two genes encoding for collagen, homologues of COL4A1 and COL4A2, the major protein of the muscle fibers [134], and a muscle LIM protein Mlp84B homolog, which is a component of the Zlines and important for maintaining the muscles integrity [135], were down-regulated during manipulated biting (Additional file 10, sheet 5 ). These suggest a mechanism by which the fungus establishes the host muscle atrophy that is important for the lock-jaw and, consequently, long term fixation of the dead ant to a plant substrate. These genes, together with the others mentioned above, which are involved in host pathways that are possible hallmarks for $O$. unilateralis s.l. to establish the different aspects of behavioral manipulation, are summarized in Table 3 and Fig. 4.

\section{Conclusions}

Given the novelty and precise nature of the behavioral changes observed, unraveling the genetic basis of fungal control of ant behavior is of great interest to the field studying parasitic manipulation of host behavior, as well as disciplines ranging from parasitology and animal behavior to parasite-host co-evolution and fungal genetics. Moreover, the long track record of fungi producing a wealth of industrially interesting bioactive compounds and the expectation that brain-manipulating species produce novel neuromodulators [41], make genetic studies into this system important from an applied perspective (both in medicine and insect pest control). Here, we used a novel O. unilateralis s.l. genome and the published C. floridanus genome as references to assess the gene expression in the heads of infected Carpenter ants during manipulated biting behavior.

Comparing the novel O. unilateralis s.l. genome to other currently available fungal genomes revealed that this manipulative fungus putatively secretes an array of novel compounds that might have interesting biological properties. Moreover, $80 \%$ of the O. unilateralis s.l. genes that were uniquely up-regulated during manipulated biting behavior were found not to be conserved among other nonmanipulating insect pathogens of the order Hypocreales. This indicates that, in order to establish manipulated biting, O. unilateralis s.l. employs a set of genes that is not present in the genomes of non-manipulating fungi. However, O. unilateralis s.l. seemingly has a mechanism to change behavior in common with a viral manipulator (i.e., $p t p$ expression). Future comparisons with other closely related (Ophiocordyceps) and non-related manipulating 
Table 3 Candidate C. castaneus targets of behavioral manipulation

\begin{tabular}{|c|c|c|}
\hline Protein ID & Functional annotation & $\begin{array}{l}\text { Fold change during } \\
\text { manipulation }\end{array}$ \\
\hline \multicolumn{3}{|l|}{ Up-regulated } \\
\hline Camfl1|CFLO16350-PA & $\begin{array}{l}\text { Putative protein-tyrosine phosphatase, involved in ELA behavior induction in Lepidoptera by } \\
\text { baculoviruses }\end{array}$ & 8.39 \\
\hline Camfl1|CFLO14092-PA & $\begin{array}{l}\text { Octopamine receptor Beta3r homolog, putative biogenic amine receptor involved in the } \\
\text { G-protein pathway }\end{array}$ & 6.26 \\
\hline Camfl1|CFLO22298-PA & $\begin{array}{l}\text { Putative specificity protein-3 transcription factor, pro-apoptotic function, also found in } \\
\text { Alzheimer brains }\end{array}$ & 5.74 \\
\hline Camfl1|CFLO15698-PA & Feminization-1 homolog, pro-apoptotic function, protein binding function & 3.14 \\
\hline Camfl1|CFLO22839-PA & $\begin{array}{l}\text { Putative protein-tyrosine phosphatase, involved in ELA behavior induction in Lepidoptera } \\
\text { by baculoviruses }\end{array}$ & 3.82 \\
\hline Camfl1|CFLO22748-PA & $\begin{array}{l}\text { Putative tyrosine 3-monooxygenase, circadian, dopamine biosynthesis and regulation of } \\
\text { locomotion behavior }\end{array}$ & 2.96 \\
\hline Camfl1|CFLO17010-PA & $\begin{array}{l}\text { Putative protein-tyrosine phosphatase, involved in ELA behavior induction in Lepidoptera } \\
\text { by baculoviruses }\end{array}$ & 2.88 \\
\hline Camfl1|CFLO19262-PA & $\begin{array}{l}\text { Putative protein-tyrosine phosphatase, involved in ELA behavior induction in Lepidoptera } \\
\text { by baculoviruses }\end{array}$ & 2.44 \\
\hline Camfl1|CFLO16807-PA & Period homolog, circadian clock gene, negative feedback loop & 2.25 \\
\hline Camfl1|CFLO21782-PA & $\begin{array}{l}\text { Putative protein-tyrosine phosphatase, involved in ELA behavior induction in Lepidoptera } \\
\text { by baculoviruses }\end{array}$ & 2.18 \\
\hline Camfl1|CFLO22839-PA & Cycle homolog, circadian clock positive feedback loop & 2.11 \\
\hline \multicolumn{3}{|l|}{ Down-regulated } \\
\hline Camfl1|CFLO13023-PA & Putative prophenoloxidase, oxidoreductase activity & 24.03 \\
\hline Camfl1|CFLO15551-PA & Putative general odorant binding protein involved in chemosignaling & 10.00 \\
\hline Camfl1|CFLO13952-PA & $\begin{array}{l}\text { Dynamin homolog, putative antimicrobial peptide, GTPase activity involved in immune } \\
\text { response }\end{array}$ & 8.08 \\
\hline Camfl1|CFLO13270-PA & Putative pheromone-binding protein involved in chemosignaling & 7.74 \\
\hline Camfl1|CFLO16107-PA & Defensin1 homolog, putative antimicrobial peptide, immune response & 5.35 \\
\hline Camfl1|CFLO17717-PA & Defensin2 homolog, putative antimicrobial peptide, immune response & 4.66 \\
\hline Camfl1|CFLO22173-PA & Odorant receptor or-1 homolog involved in chemosignaling & 4.37 \\
\hline Camfl1|CFLO15004-PA & Putative odorant receptor co-receptor involved in chemosignaling & 4.03 \\
\hline Camfl1|CFLO16950-PA & $\begin{array}{l}\text { Cytochrome p450 } 6 \mathrm{k} 1 \text { homolog, oxidoreductase activity, ion binding, involved in } \\
\text { immune response }\end{array}$ & 3.63 \\
\hline Camfl1|CFLO15757-PA & Putative collagen alpha-2 (IV) chain, muscle related & 3.19 \\
\hline Camfl1|CFLO17070-PA & Putative odorant binding protein involved in chemosignaling & 2.73 \\
\hline Camfl1|CFLO21048-PA & Muscle LIM protein Mlp84B homolog & 2.30 \\
\hline Camfl1|CFLO23127-PA & Putative peptidoglycan recognition protein involved in catabolic processes and pathogen recognition & 2.29 \\
\hline Camfl1|CFLO21233-PA & Putative lipocalin involved in stress responses & 2.23 \\
\hline Camfl1|CFLO15749-PA & Putative collagen alpha-1 (IV) chain, muscle related & 2.12 \\
\hline
\end{tabular}

parasites will therefore be interesting to investigate if the ability to manipulate host behavior has either convergently or divergently evolved.

Our study also shows that O. unilateralis s.l. has an abundance of genes encoding for bacterial-like enterotoxins, which are dynamically up- and down-regulated in the situations tested in this study. These compounds could negatively affect the production of chemosignaling molecules in insects $[31,32]$, which would impair communication in infected individuals. In agreement with this, infected ants are generally observed to be unresponsive to external stimuli $[10,60]$ and host genes putatively encoding for receptors and binding proteins involved in odorant and gustatory perception were found to be down-regulated. However, the dynamic expression of putative O. unilateralis s.l. enterotoxins could also be contributing to the up-regulated apoptotic features found in manipulated $C$. castaneus ants 
and the severe atrophy of muscle cells observed in histology studies [10].

Genes putatively involved in immune- and stress responses were found to be generally down-regulated in infected ant heads during the manipulated biting event. In fact, oxidoreductase activity-related genes were enriched in the subset of down-regulated ant genes. Oxidation-reduction reactions are vital mechanisms in parasite-host interactions for both parasite and host [73]. As such, in the fungal parasite, we indeed found genes involved in these processes being up-regulated during manipulation and down-regulated again after the host was presumably killed. While O. unilateralis s.l. ultimately kills its host, premature death will not lead to manipulation [12] thus impairing transmission and completion of the life cycle. As suggested for other insectparasite interactions [100], suppression of host responses might therefore be crucial to create conditions in the host that favor parasite development. In addition to causing apoptosis and impairing chemosensory communication, $O$. unilateralis s.l. might therefore be regulating the host's immune- and neuronal stress responses as a mechanism to establish manipulated behavior.

Other links between the fungal parasite and ant host transcriptomes during manipulated biting behavior are the up-regulation of genes encoding for enzymes involved in dopamine metabolism and protein-tyrosine phosphatases. Both pathways have reported effects on behavioral outputs such as locomotion and mandible movement, which are hallmarks of ant infections by $O$. unilateralis s.l. fungi. Moreover, homologs of clock genes per and cycle were up-regulated in manipulated ants, which would affect processes in the host, ranging from the cellular to the behavioral output level. In addition, we found the up-regulation of various fungal genes involved in different types of alkaloid metabolism that lead to compounds that can both work as agonists and antagonists of a range of receptors in the central and peripheral nervous system. Together with the up-regulation of a putatively secreted sphingomyelinase that changes neuron cell membranes, a possible shift in tryptophan metabolism from the serotonin pathway towards the kynurenine pathway and the production of NRPSs, PKSs and small secreted proteins with unknown functions, this represents an array of mechanisms and compounds through which the ant host brain could be manipulated.

In conclusion, we found various candidate manipulators and possible host pathways through which manipulated biting behavior as seen in O. unilateralis s.l.infected Carpenter ants could be established. While some of these candidates could be essential to the process, we expect these to work in concert since the manipulated behavior in this system is complex and precise. Future functional gene expression studies are necessary to determine this. Furthermore, this study only assesses the ultimate time points in this parasitehost interaction: manipulated biting and killing of the host afterwards. This leaves the mechanisms underlying the reactions and more subtle behavioral changes leading up to this still unresolved. That said, our study demonstrates that experimental infections combined with behavioral studies to determine sampling points followed by mixed transcriptomics analyses will be a good approach to study the parasite-host interactions at these earlier stages as well.

\section{Methods}

\section{Fungal strain and maintenance}

O. unilateralis s.l. strain SC16a isolated from an infected C. castaneus worker ant collected in South Carolina, USA was selected for genome sequencing and RNA-Seq since infection and manipulation of host behavior by this fungus was successfully recapitulated in the laboratory [12]. Fungal cultures were maintained in Grace's insect medium (Sigma) freshly supplemented with $10 \%$ fetal bovine serum (PAA Laboratories Inc.). For both genome sequencing and RNA-Seq, fungal cultures were grown in $250 \mathrm{~mL}$ shaking flasks holding $50 \mathrm{~mL}$ supplemented insect medium shaken at $150 \mathrm{rpm}$ at $28{ }^{\circ} \mathrm{C}$. Cultures used for infections were transferred to potato dextrose agar prior to preparing the material for injection.

\section{Genome sequencing and assembly}

For genome sequencing two different genomic libraries were prepared to avoid fragmentation bias. The first library was prepared with a Nextera kit (Illumina) according to manufacturer's instructions. For the second library $5 \mu \mathrm{g}$ of genomic DNA were sheared in a Covaris M220 ultrasonicator with $50 \mu \mathrm{l}$ screw caps to an approximate length of $800 \mathrm{bp}$. The fragmented DNA was used for library preparation with the NEBNext DNA Library Prep Master Mix Set for Illumina (New England Biolabs) according to manufacturer's instructions. Both libraries were automatically size selected to a final length of 650 850 bp by separation on a $1.5 \%$ agarose gel in a BluePippin electrophoresis unit (Sage Science). Sequencing was performed with a MiSeq sequencer (Illumina) yielding in total 13.4 Mio $2 \times 300$ bp paired-end sequences (1.4 Mio sequences for the Nextera library and 12.0 Mio sequences for the NEBNext library). Sequences were de novo assembled with CLC Genomics Workbench (Qiagen) using default parameters. $98 \%$ of primary sequences assembled into 7,875 scaffolds (minimum length $300 \mathrm{bp}$, average coverage of 120 -fold) with a total genome size of 26.05 Mb. This Whole Genome Shotgun project has been deposited at DDBJ/EMBL/GenBank under the accession LAZP00000000. The version described in this paper is version LAZP01000000. Repetitive sequences in the assembly 
were masked using RepeatMaker [136], RepBase library [137] and RepeatScout [138].

\section{Gene prediction}

Genes were predicted for $O$. unilateralis with Augustus version 3.0.2 [139] using the supplied parameter set for Fusarium graminearum. To aid in gene prediction, intron hints were produced from the Tophat transcript alignments (described below) using the Augustus software package. Introns were subsequently filtered for having at least 20 reads mapping across it and (in the case of alternative splicing) spliced in at least $50 \%$ of the cases. Completeness of the set of predicted proteins was analyzed using the CEGMA protein set [25] using a blastp E-value cutoff of 1e-5.

\section{Functional annotation}

The predicted proteins of $O$. unilateralis and those of the previously published C. floridanus [23] were functionally annotated. In addition to these, we included 16 previously published fungal genomes for comparative purposes: Ophiocordyceps sinensis [140], Tolypocladium inflatum [27], Cordyceps militaris [141], Cordyceps bassiana (formerly Beauveria bassiana) [30], Metarhizium robertsii (formerly Metarhizium anisopliae) and Metarhizium acridum [142], Claviceps purpurea [143], Fusarium oxysporum [144], Fusarium graminearum [145], Trichoderma reesei [146], Trichoderma virens [147], Magnaporthe oryzae (formerly Magnaporthe grisea) [148], Candida albicans [149], Aspergillus fumigatus [150], Neurospora crassa [151], Aspergillus nidulans [152].

Conserved protein domains were predicted using PFAM version 27 [26] and were subsequently mapped to the corresponding gene ontology (GO) terms [153, 154]. Metabolic genes were predicted using KEGG on the KAAS server [155]. Proteases were predicted using the MEROPS database [156] with a blastp E-value cutoff of 1e-5. Secretion signals were predicted using Signalp 4.1 [157]. Transmembrane domains were predicted using TMHMM 2.0c [158]. Proteins with a secretion signal, no transmembrane domain (except in the first 40 amino acids) and a total length shorter than 300 amino acids were considered small secreted proteins. Genes and gene clusters involved in secondary metabolism were predicted using a pipeline based on the SMURF method [159]. SMURF parameter $d$ (maximum intergenic distance in base pairs) was set at $3000 \mathrm{bp}$, and SMURF parameter $y$ (the maximum number of non-secondary metabolism genes upstream or downstream of the backbone gene) was set at 6 .

\section{Comparative genomic analysis}

Clusters of orthologous genes among the fungal genomes were identified using OrthoMCL version 2.0.9 [160] using an inflation factor of 1.5. To estimate the phylogeny of these organisms, 168 orthologous groups of genes having exactly one gene in each organism were identified. The sequences of each species were concatenated, aligned using MAFFT version 7.123b [161] and well-aligned regions were extracted using Gblocks 0.91b [162]. This resulted in 73994 amino acid positions. The parallelized version of RAxML version 8.1.16 [163] with the PROTGAMMAWAG model with 100 rapid bootstrap partitions was used to reconstruct a species tree. The tree was visualized using Dendroscope version 3.2.10 [164].

Proteins that contained a 'heat-labile enterotoxin alpha chain' domain (PFAM domain PF01375) were identified as enterotoxin. The domains were aligned using MAFFT version 7.123b [161]. Next, a phylogenetic tree was reconstructed with FastTree 2.0 using default parameters [165]. The tree was visualized using Dendroscope version 3.2.10 [164] and rooted on the monophyletic group containing the sequences from M. oryzae.

\section{Ant host infections and behavioral observations}

Worker ants of the species C. castaneus obtained in SC, USA (private property in Due West (GPS 34.332110, -82.387131)) were used for infections followed by RNASeq. In nature, this species is also infected by the fungal parasite used in this study. Ants were infected or sham treated by ways of injecting as previously described [12]. After the infection procedure, the colony was housed in a $513 \mathrm{~cm}^{2}$ cage with a darkened, two-dimensional $140 \mathrm{~cm}^{2}$ wooden nest and sand at the bottom. Additionally, the cage held a climbing and biting platform as well as water and $10 \%$ sugar water ad libitum. Ants were kept under strict light (LD1212) and temperature cycles with light between 06:00 $\mathrm{h}$ and $18: 00 \mathrm{~h}$ and a $10{ }^{\circ} \mathrm{C}$ increased temperature between 10:00 $\mathrm{h}$ and 16:00 $\mathrm{h}$ [12]. Ants were randomly assigned to a treatment group and color-coded (Edding): 50 untreated (no color), 35 infected (purple thorax - injected with the fungus) and 15 sham treated (green thorax injected with medium). Daily observations were made at 9:00 $\mathrm{h}, 13: 00 \mathrm{~h}$ and 17:00 $\mathrm{h}$ up to 30 days post infections to record survival status, location within the cage and the presence of manipulated climbing and biting behavior of individuals from each of the treatment groups.

\section{Preparation of controls and infected samples}

To reduce variation within sample type, we used heads of ants that were collected on the same day. The maximum number found manipulated on the same day was three. Therefore, each of the sample types was prepared in biological triplicates. O. unilateralis s.l. controls were generated from saturated shaking cultures that were grown for 14 days. These cultures were harvested over a sterile Buchner funnel with Whatmann paper using suction. Dried mycelium was subsequently snap frozen in liquid nitrogen and stored at $-80{ }^{\circ} \mathrm{C}$. Healthy C. castaneus 
controls were collected after keeping them under the same strict $24 \mathrm{~h}$ cycles as the infected ants for 30 days. The day prior to sampling, 6 untreated ants were divided over 2 small cages placed next to the cage used for the infection experiment to be able to snap freeze them in liquid nitrogen without inducing stress due to sampling. At 10:00 h 3 ants were harvested by quickly inverting one small cage into liquid nitrogen. These ants function as controls for infected ants displaying manipulated biting behavior. At 14:00 $\mathrm{h}$ the 3 additional ants were harvested to function as controls for ants that died after the manipulated biting event. Infected ants displaying the manipulated biting behavior were harvested at 10:00 $\mathrm{h}$ by placing them, together with the twig they were biting, into liquid nitrogen. Similarly, infected ants that appeared lifeless after biting were harvested at 14:00 h. Manipulated ants, not reacting to any environmental stimuli anymore, would not be disturbed by the sampling procedure. After snap freezing, twigs were removed and harvested ants were stored at $-80{ }^{\circ} \mathrm{C}$ prior to RNA extraction.

\section{RNA extraction, mRNA-Seq library construction and sequencing}

RNA was extracted from $1 \mathrm{~cm}^{2}$ pieces of the $O$. unilateralis control cultures, and the heads from both the infected and the healthy control ants. These samples were transferred to liquid nitrogen cooled $2 \mathrm{~mL}$ Eppendorf tubes (Greiner) with two metal beads $(4.76 \mathrm{~mm}$ in diameter). Cells were mechanically disrupted inside these frozen $2 \mathrm{~mL}$ Eppendorf using a TissueLyser II (Qiagen) and a chilled adapter set (Qiagen) at $24 \mathrm{freq} / \mathrm{s}$ for $60 \mathrm{~s}$. RNA was subsequently extracted according to a previously reported protocol [166]. The quality and quantity of the samples were checked using a Bioanalyzer (Agilent Technologies) and a NanoDrop (Thermo Scientific). Subsequently mRNA-Seq libraries were constructed using a TruSeq Stranded mRNA Sample Prep Kit (Illumina). Samples were sequenced on an Illumina HiSeq 2500 using $100 \times 100$ paired-end sequencing in Rapid Run mode. The RNA-Seq expression dataset is available at the NCBI's Gene Expression Omnibus under the accession code GSE68176.

\section{Mixed transcriptome analysis}

Both single species RNA reads (as controls for both parasite and host) and mixed RNA reads (in case of parasite-host interactions) were used in this study. Sequence tags were mapped to the respective parasite and host genome sequences using the programs TopHat version 2.0.11 [167] and Bowtie version 2.2.2 [168]. Default settings were used, with the exception of intron length, which was set as a minimum of 5 nucleotides and a maximum of 1000 nucleotides in the case of $O$. unilateralis. Moreover, the microexon-search option was enabled. The program Cuffdiff version 2.2.1, which is part of Cufflinks [169], was used to identify reads overlapping with the predicted genes and to identify differentially expressed genes. Default settings were used. The expression levels of each predicted gene were normalized to Fragments Per Kilobase of exon model per Million fragments (FPKM). The bias correction method was used while running Cuffdiff [170]. For differential expression we also applied a cutoff of at least a 2 -fold change in expression value, as well as at least one condition with an expression value of at least 4 FPKM. Over- and under-representation of functional annotation terms in sets of differentially regulated genes were calculated using the Fisher Exact test. The Benjamini-Hochberg correction was used to correct for multiple testing. As a cutoff for significance we used a corrected $p$-value of 0.05 . To visualize gene expression a heat map was generated using thw 'heatmap.2' module of the statistics software package R 3.2.0. First, the expression values were log2-transformed after increasing the values with 1 in order to avoid negative $\log 2$ values. Next, the genes were clustered using the euclidean distance and average linkage methods. The values were scaled for each gene, resulting in a z-score.

\section{Additional files}

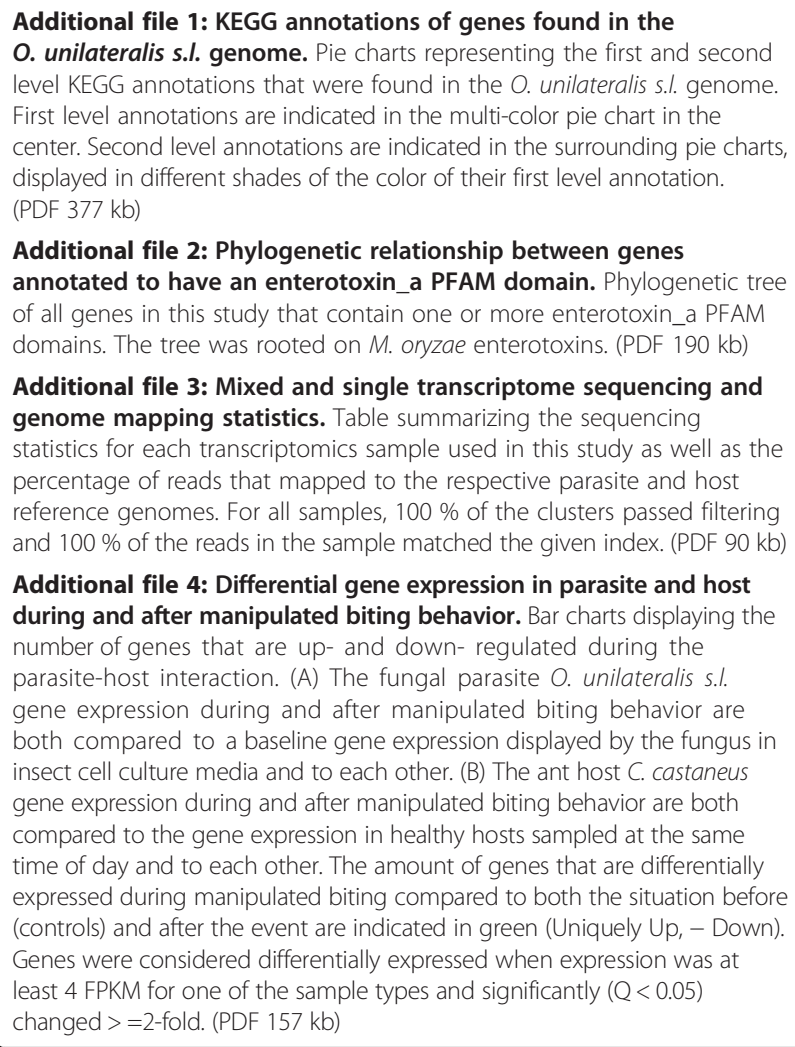
displayed in different shades of the color of their first level annotation. (PDF 377 kb)

Additional file 2: Phylogenetic relationship between genes annotated to have an enterotoxin_a PFAM domain. Phylogenetic tree of all genes in this study that contain one or more enterotoxin_a PFAM domains. The tree was rooted on M. oryzae enterotoxins. (PDF $190 \mathrm{~kb}$ )

Additional file 3: Mixed and single transcriptome sequencing and genome mapping statistics. Table summarizing the sequencing statistics for each transcriptomics sample used in this study as well as the percentage of reads that mapped to the respective parasite and host reference genomes. For all samples, $100 \%$ of the clusters passed filtering and $100 \%$ of the reads in the sample matched the given index. (PDF $90 \mathrm{~kb}$ )

Additional file 4: Differential gene expression in parasite and host during and after manipulated biting behavior. Bar charts displaying the number of genes that are up- and down- regulated during the parasite-host interaction. (A) The fungal parasite O. unilateralis s.l. gene expression during and after manipulated biting behavior are both compared to a baseline gene expression displayed by the fungus in insect cell culture media and to each other. (B) The ant host $C$. castaneus gene expression during and after manipulated biting behavior are both compared to the gene expression in healthy hosts sampled at the same time of day and to each other. The amount of genes that are differentially expressed during manipulated biting compared to both the situation before (controls) and after the event are indicated in green (Uniquely Up, - Down). Genes were considered differentially expressed when expression was at least 4 FPKM for one of the sample types and significantly $(Q<0.05)$ changed $>=2$-fold. (PDF $157 \mathrm{~kb}$ ) 
Additional file 5: Mapped fungal and insect reads in mixed transcriptome samples compared to single transcriptome controls. Bar charts displaying the percentage of RNA-Seq reads in both single and mixed transcriptome samples mapped to either the parasitic O. unilateralis s.l. genome (A) or the C. floridanus genome (B). The single transcriptome samples (Fungal Culture and Healthy Ant heads; black bars) display the read mapping when all of the biological material is derived from one organism. Relative comparison to this percentage reads mapped suggests that during manipulated biting behavior about half of the biological material is ant tissue, while the other half comprises of fungal cells. Right after the manipulated biting event the amount of fungal cells inside the head appears to have increased, while the amount of host tissue has decreased even further. (PDF $131 \mathrm{~kb}$ )

Additional file 6: Enrichment analysis on differentially expressed O. unilateralis s.l. genes. Tables summarizing the overrepresentation of annotation terms for $O$. unilateralis s.l. genes found to be up- and down-regulated during manipulated biting behavior in comparison to baseline gene expression in fungal cultures (Tables $\mathbf{S} \mathbf{1}$ and $\mathbf{S 2}$, respectively), after manipulated biting behavior in comparison to baseline gene expression (Tables S3 and S4), during manipulated biting behavior versus death after manipulated biting behavior (Tables S5 and S6), and genes that are uniquely up- or down-regulated during manipulated biting behavior (Tables S7 and S8). (PDF $263 \mathrm{~kb}$ )

Additional file 7: Differentially expressed 0 . unilateralis s.l. genes. Tables displaying all $O$. unilateralis s.l. genes, including their expression levels and annotation, that were found to be differentially expressed during manipulated biting behavior in comparison to baseline gene expression in fungal cultures (sheets 2 and 3), after manipulated biting behavior in comparison to baseline gene expression (sheets 4 and 5), and during manipulated biting behavior versus death after manipulated biting behavior (sheets 6 and 7). (XLSX $891 \mathrm{~kb}$ )

Additional file 8: $O$. unilateralis s.l. genes uniquely up- or down regulated during manipulated biting behavior. Tables displaying all O. unilateralis s.l. genes, including their expression levels and annotation that were found to be up-regulated during manipulated biting behavior followed by being down-regulated again after the biting event had taken place (sheet 2), and that were found to be down-regulated during manipulated biting behavior followed by being up-regulated again after the biting event had taken place (sheet 3). (XLSX $232 \mathrm{~kb}$ )

\section{Additional file 9: An expression heat map of differentially} expressed 0 . unilateralis s.l. genes that contain a putative secretion signal. Of 891 genes encoding a protein with a putative secretion signal, 429 (48 \%) were differentially expressed. Green indicates a relatively high expression of that gene compared to other conditions, whereas red indicates relatively low expression. Genes are clustered based on similarity of expression profile. The large differences in expression between the samples are clearly visible. Genes annotated to be small secreted proteins (SSPs) are indicated with a line in between the dendrogram and the heat map. (PDF $538 \mathrm{~kb}$ )

\section{Additional file 10: Differentially expressed C. castaneus genes.}

Tables displaying all C. castaneus genes, including their expression levels and annotation, that were found to be differentially expressed in healthy ant heads at 2 different time points during the day; 10:00 $\mathrm{h}$ and 14:00 $\mathrm{h}$ (sheets 2 and 3), during manipulated biting behavior in comparison to healthy ant heads at 10:00 $\mathrm{h}$ (sheets 4 and 5), after manipulated biting behavior in comparison to healthy ant heads at 14:00 h (sheets 6 and 7), and during manipulated biting behavior versus death after manipulated biting behavior (sheets 8 and 9). (XLSX $339 \mathrm{~kb}$ )

Additional file 11: Enrichment analysis on differentially expressed C. castaneus genes. Tables summarizing the overrepresentation of annotation terms for $C$. castaneus genes found to be up- and down-regulated when healthy ants are compared at two different times of day; $10 \mathrm{AM}$ and 2 PM (Tables S1 and S2), during manipulated biting behavior in comparison to healthy ant controls (Tables S3 and S4), after manipulated biting behavior in comparison to healthy ant controls (Tables S5 and S6), during manipulated biting behavior versus death after manipulated biting behavior (Tables S7 and S8), and genes that are uniquely up- or down-regulated during manipulated biting behavior (Tables S9 and S10). (PDF $554 \mathrm{~kb}$ )

\section{Competing interests}

The authors declare that they have no competing interests.

\section{Authors' contributions}

Conceived, designed and performed the experiments: CDB, RGL, DPH. Genome sequencing and assembly: AB. Bioinformatics: CDB, RAO, AS, IUA. Analyzed the data: CDB, RAO, RGL, DPH. Wrote paper: CDB, RAO, RGL, MM, $A B, D P H$. All authors read and approved the final manuscript.

\section{Acknowledgements}

We would like to thank the Huck Institute of the Life Sciences and the Penn State Genomics Core Facility - University Park, PA for providing us with their RNA sequencing services and guidance in choosing the right setup for this part of the project. We would like to thank Roel Fleuren from Science Transmitter for assisting us in the making of the figures for this manuscript. This work was supported by Marie Curie Actions IOF Project 299501, CAPES-Brazil project \#6203/10-8, and funds to DPH from Penn State. This project also benefitted from donations from the crowd made available to us through experiment.com/projects/how-does-a-parasite-create-zombie-like-behavior. We therefore would like to thank the backers of this project for their support since their contributions greatly helped us in generating the datasets presented in this paper.

\section{Author details}

'Institute of Medical Psychology, Faculty of Medicine,

Ludwig-Maximilians-University Munich, Goethestrasse 31, 80336 Munich, Germany. ${ }^{2}$ Department of Entomology and Department of Biology, Center for Infectious Disease Dynamics, Pennsylvania State University, University Park, State College, Pennsylvania 16802 PA, USA. ${ }^{3}$ Microbiology, Faculty of Science, Utrecht University, Padualaan 8, 3584 CH, Utrecht, The Netherlands. ${ }^{4}$ CAPES Foundation, Ministry of Education of Brazil, Brasília 70040-020 DF, Brazil. ${ }^{5}$ Bioinformatics Consulting Center, Pennsylvania State University, University Park, State College, Pennsylvania 16802 PA, USA. ${ }^{6}$ Department of Biochemistry and Molecular Biology, The Huck Institutes of the Life Sciences, Pennsylvania State University, University Park, State College, Pennsylvania 16802 PA, USA. ${ }^{7}$ Faculty of Biology, Section Genetics,

Ludwig-Maximilians-University Munich, Grosshaderner Strasse 2-4, 82152 Martinsried, Germany.

Received: 24 June 2015 Accepted: 3 August 2015 Published online: 19 August 2015

\section{References}

1. Moore J. Parasites and the behavior of animals. Oxford series in ecology and evolution. Oxford, New York: Oxford University Press; 2002.

2. Thomas F, Adamo S, Moore J. Parasitic manipulation: where are we and where should we go? Behav Process. 2005;68:185-99.

3. Hughes DP, Brodeur J, Thomas F. Host manipulation by parasites. Oxford, New York: Oxford University Press; 2012.

4. Dawkins R. The extended phenotype. Oxford: W.H. Freeman; 1982.

5. Kamita SG, Nagasaka K, Chua JW, Shimada T, Mita K, Kobayashi M, et al. A baculovirus-encoded protein tyrosine phosphatase gene induces enhanced locomotory activity in a lepidopteran host. Proc Natl Acad Sci U S A. 2005;102:2584-9.

6. Lefèvre T, Adamo SA, Biron DG, Misse D, Hughes DP, Thomas F. Invasion of the body snatchers: the diversity and evolution of manipulative strategies in host-parasite interactions. Adv Parasit. 2009;68:45-83.

7. Lefèvre T, Lebarbenchon C, Gauthier-Clerc M, Misse D, Poulin R, Thomas F. The ecological significance of manipulative parasites. Trends Ecol Evol. 2009;24:41-8.

8. Libersat F, Delago A, Gal R. Manipulation of host behavior by parasitic insects and insect parasites. Annu Rev Entomol. 2009;54:189-207.

9. Hoover K, Grove M, Gardner M, Hughes DP, McNeil J, Slavicek J. A gene for an extended phenotype. Science. 2011;333:1401.

10. Hughes DP, Andersen SB, Hywel-Jones NL, Himaman W, Billen J, Boomsma $J$ J. Behavioral mechanisms and morphological symptoms of zombie ants dying from fungal infection. BMC Ecol. 2011;11:13.

11. van Houte S, Ros VI, Mastenbroek TG, Vendrig NJ, Hoover K, Spitzen J, et al. Protein tyrosine phosphatase-induced hyperactivity is a conserved strategy of a subset of baculoviruses to manipulate lepidopteran host behavior. PLoS One. 2012;7, e46933. 
12. de Bekker C, Quevillon LE, Smith PB, Fleming KR, Ghosh D, Patterson AD, et al. Species-specific ant brain manipulation by a specialized fungal parasite. BMC Evol Biol. 2014;14:166

13. Smirnoff WA. Observations on the effect of virus infection on insect behavior. J Invertebr Pathol. 1965;7:387-8.

14. Evans HF. Ecology and epizootiology of baculoviruses. In: Granados RRF BA, editor. The biology of baculoviruses. Boca Raton, FL: CRC Press; 1986. p. 89-132.

15. Goulson D. Wipfelkrankheit: modification of host behaviour during baculoviral infection. Oecologia. 1997;109:219-28.

16. Nijhout HF. Insect hormones. Princeton: Princeton University Press; 1994.

17. van Houte $S$, van Oers MM, Han Y, Vlak JM, Ros VI. Baculovirus infection triggers a positive phototactic response in caterpillars to induce 'tree-top' disease. Biol Lett. 2014;10:20140680.

18. Andersen SB, Gerritsma S, Yusah KM, Mayntz D, Hywel-Jones NL, Billen J, et al. The life of a dead ant: the expression of an adaptive extended phenotype. Am Nat. 2009;174:424-33.

19. Evans HC. Entomogenous fungi in the tropical forest ecosystems: an appraisal. Ecol Entomol. 1982;7:47-60.

20. Evans HC, Samson RA. Cordyceps species and their anamorphs pathogenic on ants (Formicidae) in tropical forest ecosystems. II. The Camponotus (Formicinae) complex. T Brit Mycol Soc. 1984;82:127-50.

21. Pontoppidan M-B, Himaman W, Hywel-Jones NL, Boomsma JJ, Hughes DP. Graveyards on the move: the spatio-temporal distribution of dead Ophiocordyceps-infected ants. PLoS One. 2009;4, e4835.

22. Andersen SB, Ferrari M, Evans HC, Elliot SL, Boomsma JJ, Hughes DP. Disease dynamics in a specialized parasite of ant societies. PLoS One. 2012;7, e36352.

23. Bonasio R, Zhang GJ, Ye CY, Mutti NS, Fang XD, Qin N, et al. Genomic comparison of the ants Camponotus floridanus and Harpegnathos saltator. Science. 2010;329:1068-71.

24. Parra G, Bradnam K, Korf I. CEGMA: a pipeline to accurately annotate core genes in eukaryotic genomes. Bioinformatics. 2007;23:1061-7.

25. Parra G, Bradnam K, Ning Z, Keane T, Korf I. Assessing the gene space in draft genomes. Nucleic Acids Res. 2009;37:289-97.

26. Finn RD, Bateman A, Clements J, Coggill P, Eberhardt RY, Eddy SR, et al. Pfam: the protein families database. Nucleic Acids Res. 2014;42:D222-30.

27. Bushley KE, Raja R, Jaiswal P, Cumbie JS, Nonogaki M, Boyd AE, et al. The genome of Tolypocladium inflatum: evolution, organization, and expression of the cyclosporin biosynthetic gene cluster. PLoS Genet. 2013;9, e1003496.

28. Martin F, Aerts A, Ahren D, Brun A, Danchin EG, Duchaussoy F, et al. The genome of Laccaria bicolor provides insights into mycorrhizal symbiosis. Nature. 2008;452:88-92.

29. Ohm RA, Feau N, Henrissat B, Schoch CL, Horwitz BA, Barry KW, et al. Diverse lifestyles and strategies of plant pathogenesis encoded in the genomes of eighteen Dothideomycetes fungi. PLoS Pathog. 2012;8, e1003037.

30. Xiao G, Ying SH, Zheng P, Wang ZL, Zhang S, Xie XQ, et al. Genomic perspectives on the evolution of fungal entomopathogenicity in Beauveria bassiana. Sci Rep. 2012;2:483.

31. Wiygul G, Sikorowski PP. The effect of staphylococcal enterotoxin B on pheromone production in fat bodies isolated from male boll weevils. J Invertebr Pathol. 1986:47:116-9.

32. Wiygul G, Sikorowski PP. The effect of a heat-stable enterotoxin isolated from Escherichia coli on pheromone production in fat bodies isolated from male boll weevils. Entomol Exp Appl. 1991;60:305-8.

33. Hölldobler B, Wilson EO. The ants. Cambridge: Mass.: Harvard University Press; 1990.

34. Claridge-Chang A, Wijnen H, Naef F, Boothroyd C, Rajewsky N, Young MW. Circadian regulation of gene expression systems in the Drosophila head. Neuron. 2001;32:657-71.

35. Yek SH, Boomsma JJ, Schiott M. Differential gene expression in Acromyrmex leaf-cutting ants after challenges with two fungal pathogens. Mol Ecol. 2013;22:2173-87.

36. de Bekker C, Smith PB, Patterson AD, Hughes DP. Metabolomics reveals the heterogeneous secretome of two entomopathogenic fungi to ex vivo cultured insect tissues. PLoS One. 2013;8, e70609.

37. Črešnar B, Petrič S. Cytochrome P450 enzymes in the fungal kingdom. Biochim Biophys Acta. 1814;2011:29-35.

38. Panstruga $R$, Dodds PN. Terrific protein traffic: the mystery of effector protein delivery by filamentous plant pathogens. Science. 2009;324:748-50.
39. Stergiopoulos I, de Wit PJ. Fungal effector proteins. Annu Rev Phytopathol. 2009;47:233-63.

40. Biron DG, Loxdale HD. Host-parasite molecular cross-talk during the manipulative process of a host by its parasite. J Exp Biol. 2013;216:148-60.

41. Molnár I, Gibson DM, Krasnoff SB. Secondary metabolites from entomopathogenic Hypocrealean fungi. Nat Prod Rep. 2010;27:1241-75.

42. Heinstein PF, Lee SI, Floss HG. Isolation of dimethylallylpyrophosphate: tryptophan dimethylallyl transferase from the ergot fungus (Claviceps spec.). Biochem Biophys Res Commun. 1971:44:1244-51.

43. Tsai HF, Wang H, Gebler JC, Poulter CD, Schardl CL. The Claviceps purpurea gene encoding dimethylallyltryptophan synthase, the committed step for ergot alkaloid biosynthesis. Biochem Biophys Res Co. 1995;216:119-25

44. Schardl CL, Panaccione DG, Tudzynski P. Ergot alkaloids-biology and molecular biology. Alkaloids Chemistry Biol. 2006;63:45-86.

45. Eadie MJ. Convulsive ergotism: epidemics of the serotonin syndrome? Lancet Neurol. 2003;2:429-34.

46. Dunn MF, Niks D, Ngo H, Barends TR, Schlichting I. Tryptophan synthase: the workings of a channeling nanomachine. Trends Biochem Sci. 2008;33:254-64.

47. Young C, McMillan L, Telfer E, Scott B. Molecular cloning and genetic analysis of an indole-diterpene gene cluster from Penicillium paxilli. Mol Microbiol. 2001;39:754-64.

48. Tagami K, Liu C, Minami A, Noike M, Isaka T, Fueki S, et al. Reconstitution of biosynthetic machinery for indole-diterpene paxilline in Aspergillus oryzae. J Am Chem Soc. 2013;135:1260-3.

49. Saikia S, Parker EJ, Koulman A, Scott B. Four gene products are required for the fungal synthesis of the indole-diterpene, paspaline. FEBS Lett. 2006:580:1625-30.

50. Xu W, Gavia DJ, Tang Y. Biosynthesis of fungal indole alkaloids. Nat Prod Rep. 2014;31:1474-87.

51. Parker EJS B. Indole-diterpene biosynthesis in ascomycetous fungi. In: An Z editor. Handbook of industrial Mycology. New York, NY: Taylor \& Francis; 2004. p. 405-26

52. Saikia S, Nicholson MJ, Young C, Parker EJ, Scott B. The genetic basis for indole-diterpene chemical diversity in filamentous fungi. Mycol Res. 2008;112:184-99.

53. Sings HS S. Tremorgenic and non-tremorgenic 2,3-fused indole diterpenoids. In: Cordell GA, editor. The alkaloids: chemistry and biology. Amsterdam, New York: Elsevier Science; 2003. p. 51-163.

54. Drevets WC, Zarate Jr CA, Furey ML. Antidepressant effects of the muscarinic cholinergic receptor antagonist scopolamine: a review. Biol Psychiat. 2013;73:1156-63.

55. Heyes MP, Saito K, Crowley JS, Davis LE, Demitrack MA, Der M, et al. Quinolinic acid and kynurenine pathway metabolism in inflammatory and non-inflammatory neurological disease. Brain: J Neurol. 1992;115 (Pt 5):1249-73.

56. Schwarcz R, Whetsell Jr WO, Mangano RM. Quinolinic acid: an endogenous metabolite that produces axon-sparing lesions in rat brain. Science. 1983;219:316-8.

57. Heyes MP, Lackner A. Increased cerebrospinal fluid quinolinic acid, kynurenic acid, and L-kynurenine in acute septicemia. J Neurochem. 1990;55:338-41.

58. Vamos E, Pardutz A, Klivenyi P, Toldi J, Vecsei L. The role of kynurenines in disorders of the central nervous system: possibilities for neuroprotection. J Neurol Sci. 2009;283:21-7.

59. Muscedere ML, Johnson N, Gillis BC, Kamhi JF, Traniello JF. Serotonin modulates worker responsiveness to trail pheromone in the ant Pheidole dentata. J Comp Physiol A. 2012;198:219-27.

60. Loreto RG, Elliot SL, Freitas ML, Pereira TM, Hughes DP. Long-term disease dynamics for a specialized parasite of ant societies: a field study. PLoS One. 2014;9, e103516.

61. Ganfornina MD, Kayser H, Sanchez D. Lipocalins in arthropoda: diversification and functional explorations. Madame Curie Bioscience Database. Austin, Texas: Landes Bioscience; 2000.

62. Suzuki K, Lareyre JJ, Sanchez D, Gutierrez G, Araki Y, Matusik RJ, et al. Molecular evolution of epididymal lipocalin genes localized on mouse chromosome 2. Gene. 2004;339:49-59.

63. Charron JB, Ouellet F, Pelletier M, Danyluk J, Chauve C, Sarhan F. Identification, expression, and evolutionary analyses of plant lipocalins. Plant Physiol. 2005;139:2017-28. 
64. Flower DR. Multiple molecular recognition properties of the lipocalin protein family. J Mol Recognit. 1995;8:185-95.

65. Ban L, Scaloni A, Brandazza A, Angeli S, Zhang L, Yan Y, et al. Chemosensory proteins of Locusta migratoria. Insect Mol Biol. 2003;12:125-34.

66. Calvello M, Guerra N, Brandazza A, D'Ambrosio C, Scaloni A, Dani FR, et al. Soluble proteins of chemical communication in the social wasp Polistes dominulus. Cell Mol Life Sci. 2003;60:1933-43.

67. Walker DW, Muffat J, Rundel C, Benzer S. Overexpression of a Drosophila homolog of apolipoprotein D leads to increased stress resistance and extended lifespan. Curr Biol. 2006;16:674-9.

68. Monod M, Capoccia S, Lechenne B, Zaugg C, Holdom M, Jousson O. Secreted proteases from pathogenic fungi. Int J Med Microbiol. 2002;292:405-19.

69. Khavandgar Z, Murshed M. Sphingolipid metabolism and its role in the skeletal tissues. Cell Mol Life Sci. 2015;72:959-69.

70. Stoffel W. Functional analysis of acid and neutral sphingomyelinases in vitro and in vivo. Chem Phys Lipids. 1999;102:107-21.

71. Doll VM, Ehling-Schulz M, Vogelmann R. Concerted action of sphingomyelinase and non-hemolytic enterotoxin in pathogenic Bacillus cereus. PLoS One. 2013;8.

72. Kohen R, Nyska A. Oxidation of biological systems: oxidative stress phenomena, antioxidants, redox reactions, and methods for their quantification. Toxicol Pathol. 2002;30:620-50.

73. Adak S, Pal S. Ascorbate peroxidase acts as a novel determiner of redox homeostasis in Leishmania. Antioxid Redox Sign. 2013;19:746-54.

74. Piacenza L, Peluffo G, Alvarez MN, Martinez A, Radi R. Trypanosoma cruzi antioxidant enzymes as virulence factors in Chagas disease. Antioxid Redox Sign. 2013;19:723-34

75. Werck-Reichhart D, Feyereisen R. Cytochromes P450: a success story. Genome Biol. 2000;1:REVIEWS3003.

76. Henderson B, Higgs GA. Synthesis of arachidonate oxidation products by synovial joint tissues during the development of chronic erosive arthritis. Arthritis Rheum. 1987;30:1149-56.

77. Kliewer SA, Goodwin B, Willson TM. The nuclear pregnane X receptor: a key regulator of xenobiotic metabolism. Endocr Rev. 2002;23:687-702.

78. Wang GT W, Bidigare RR. Terpenoids as therapeutic drugs and pharmaceutical agents. In: Zhang LD AL, editor. Natural Products: Drug discovery and therapeutic medicine. Totowa, NJ: Humana Press Inc; 2005. p. 197-227.

79. Sondergaard TE, Hansen FT, Purup S, Nielsen AK, Bonefeld-Jorgensen EC, Giese $\mathrm{H}$, et al. Fusarin $\mathrm{C}$ acts like an estrogenic agonist and stimulates breast cancer cells in vitro. Toxicol Lett. 2011;205:116-21.

80. Krasnoff SB, Sommers CH, Moon YS, Donzelli BG, Vandenberg JD, Churchill AC, et al. Production of mutagenic metabolites by Metarhizium anisopliae. J Agr Food Chem. 2006;54:7083-8.

81. Sanchez-Ferrer A, Rodriguez-Lopez JN, Garcia-Canovas F, Garcia-Carmona F. Tyrosinase: a comprehensive review of its mechanism. Biochim Biophys Acta. 1995;1247:1-11

82. Asanuma M, Miyazaki I, Ogawa N. Dopamine- or L-DOPA-induced neurotoxicity: the role of dopamine quinone formation and tyrosinase in a model of Parkinson's disease. Neurotox Res. 2003:5:165-76.

83. Bell AA, Wheeler MH. Biosynthesis and functions of fungal melanins. Annu Rev Phytopathol. 1986;24:411-51.

84. Jacobson ES. Pathogenic roles for fungal melanins. Clin Microbiol Rev. 2000;13:708-17.

85. Nappi AJ, Christensen BM. Melanogenesis and associated cytotoxic reactions: applications to insect innate immunity. Insect Biochem Molec. 2005;35:443-59.

86. Miyazaki I, Asanuma M. Approaches to prevent dopamine quinone-induced neurotoxicity. Neurochem Res. 2009;34:698-706.

87. Berman SB, Hastings TG. Dopamine oxidation alters mitochondrial respiration and induces permeability transition in brain mitochondria: implications for Parkinson's disease. J Neurochem. 1999;73:1127-37.

88. Kuhn DM, Francescutti-Verbeem DM, Thomas DM. Dopamine quinones activate microglia and induce a neurotoxic gene expression profile: relationship to methamphetamine-induced nerve ending damage. Ann NY Acad Sci. 2006;1074:31-41.

89. Miyazaki I, Asanuma M, Diaz-Corrales FJ, Fukuda M, Kitaichi K, Miyoshi K, et al. Methamphetamine-induced dopaminergic neurotoxicity is regulated by quinone-formation-related molecules.

FASEB J. 2006;20:571-3.
90. Zafar KS, Siegel D, Ross D. A potential role for cyclized quinones derived from dopamine, DOPA, and 3,4-dihydroxyphenylacetic acid in proteasomal inhibition. Mol Pharmacol. 2006;70:1079-86.

91. Varrot A, Basheer SM, Imberty A. Fungal lectins: structure, function and potential applications. Curr Opin Struc Biol. 2013;23:678-85.

92. Hamshou M, Van Damme EJ, Caccia S, Cappelle K, Vandenborre G, Ghesquière $B$, et al. High entomotoxicity and mechanism of the fungal GalNAc/Gal-specific Rhizoctonia solani lectin in pest insects. J Insect Physiol. 2013;59:295-305.

93. Berne S, Lah L, Sepčić K. Aegerolysins: structure, function, and putative biological role. Protein Sci. 2009;18:694-706.

94. Rementeria A, López-Molina N, Ludwig A, Vivanco AB, Bikandi J, Pontón J, et al. Genes and molecules involved in Aspergillus fumigatus virulence. Rev Iberoam Micol. 2005;22:1-23.

95. Ratzka C, Liang C, Dandekar T, Gross R, Feldhaar H. Immune response of the ant Camponotus floridanus against pathogens and its obligate mutualistic endosymbiont. Insect Biochem Molec. 2011;41:529-36.

96. Konrad M, Vyleta ML, Theis FJ, Stock M, Tragust S, Klatt M, et al. Social transfer of pathogenic fungus promotes active immunisation in ant colonies. PLOS Biol. 2012;10, e1001300

97. Silverman N, Zhou R, Erlich RL, Hunter M, Bernstein E, Schneider D, et al. Immune activation of NF-kappaB and JNK requires Drosophila TAK1. J Biol Chem. 2003;278:48928-34.

98. Ruiz M, Wicker-Thomas C, Sanchez D, Ganfornina MD. Grasshopper Lazarillo, a GPI-anchored Lipocalin, increases Drosophila longevity and stress resistance, and functionally replaces its secreted homolog NLaz. Insect Biochem Molec. 2012;42:776-89.

99. Yang $X$, Cox-Foster DL. Impact of an ectoparasite on the immunity and pathology of an invertebrate: evidence for host immunosuppression and viral amplification. Proc Natl Acad Sci U S A. 2005;102:7470-5.

100. Barribeau SM, Sadd BM, du Plessis L, Schmid-Hempel P. Gene expression differences underlying genotype-by-genotype specificity in a host-parasite system. Proc Natl Acad Sci U S A. 2014;111:3496-501.

101. Hu C, Aksoy S. Innate immune responses regulate trypanosome parasite infection of the tsetse fly Glossina morsitans morsitans. Mol Micobiol. 2006;60:1194-204.

102. Telleria EL, Sant'Anna MR, Ortigao-Farias JR, Pitaluga AN, Dillon VM, Bates PA, et al. Caspar-like gene depletion reduces Leishmania infection in sand fly host Lutzomyia longipalpis. J Biol Chem. 2012;287:12985-93.

103. Hodgkin J, Doniach T, Shen M. The sex determination pathway in the nematode Caenorhabditis elegans: variations on a theme. Cold Spring Harb Sym. 1985;50:585-93.

104. Gaudet J, VanderElst I, Spence AM. Post-transcriptional regulation of sex determination in Caenorhabditis elegans: widespread expression of the sex-determining gene fem-1 in both sexes. Mol Biol Cell. 1996;7:1107-21.

105. Chan SL, Tan KO, Zhang L, Yee KS, Ronca F, Chan MY, et al. F1Aalpha, a death receptor-binding protein homologous to the Caenorhabditis elegans sex-determining protein, FEM-1, is a caspase substrate that mediates apoptosis. J Biol Chem. 1999;274:32461-8.

106. Chan SL, Yee KS, Tan KM, Yu VC. The Caenorhabditis elegans sex determination protein FEM-1 is a CED-3 substrate that associates with CED-4 and mediates apoptosis in mammalian cells. J Biol Chem. 2000;275:17925-8

107. Essafi-Benkhadir K, Grosso S, Puissant A, Robert G, Essafi M, Deckert M, et al. Dual role of Sp3 transcription factor as an inducer of apoptosis and a marker of tumour aggressiveness. PLoS One. 2009;4, e4478.

108. Ban K, Kozar RA. Glutamine protects against apoptosis via downregulation of Sp3 in intestinal epithelial cells. Am J Physiol Gastro L. 2010;299:G1344-53.

109. Wilson AJ, Chueh AC, Togel L, Corner GA, Ahmed N, Goel S, et al. Apoptotic sensitivity of colon cancer cells to histone deacetylase inhibitors is mediated by an Sp1/Sp3-activated transcriptional program involving immediate-early gene induction. Cancer Res. 2010;70:609-20.

110. Boutillier S, Lannes B, Buee L, Dlelacourte A, Rouaux C, Mohr M, et al. Sp3 and sp4 transcription factor levels are increased in brains of patients with Alzheimer's disease. Neurodegener Dis. 2007;4:413-23.

111. Wunderlich K, Smittenaar P, Dolan RJ. Dopamine enhances model-based over model-free choice behavior. Neuron. 2012;75:418-24.

112. Yu Q, Teixeira CM, Mahadevia D, Huang Y, Balsam D, Mann JJ, et al. Dopamine and serotonin signaling during two sensitive developmental periods differentially impact adult aggressive and affective behaviors in mice. Mol Psychiatr. 2014;19:688-98. 
113. Huber R, Orzeszyna M, Pokorny N, Kravitz EA. Biogenic amines and aggression: experimental approaches in crustaceans. Brain Behav Evolut. 1997;50:60-8.

114. Riemensperger T, Isabel G, Coulom H, Neuser K, Seugne L, Kume K, et al. Behavioural consequences of dopamine deficiency in the Drosophila brain. J Neurogenet. 2010;24:16.

115. McQuillan HJ, Barron AB, Mercer AR. Age- and behaviour-related changes in the expression of biogenic amine receptor genes in the antennae of honey bees (Apis mellifera). J Comp Physiol A. 2012;198:753-61.

116. Sitaraman D, LaFerriere $H$, Birman S, Zars T. Serotonin is critical for rewarded olfactory short-term memory in Drosophila. J Neurogenet. 2012;26:238-44.

117. Fossat $P$, Bacqué-Cazenave J, De Deurwaerdère $P$, Delbecque JP, Cattaert $D$. Comparative behavior anxiety-like behavior in crayfish Is controlled by serotonin. Science. 2014;344:1293-7.

118. Kamhi JF, Traniello JFA. Biogenic amines and collective organization in a superorganism: Neuromodulation of social behavior in ants. Brain Behav Evolut. 2013;82:220-36

119. Smith AR, Muscedere ML, Seid MA, Traniello JFA, Hughes WOH. Biogenic amines are associated with worker task but not patriline in the leaf-cutting ant Acromyrmex echinatior. J Comp Physiol A. 2013;199:1117-27.

120. Szczuka A, Korczyńska J, Wnuk A, Symonowicz B, Gonzalez Szwacka A, Mazurkiewicz $P$, et al. The effects of serotonin, dopamine, octopamine and tyramine on behavior of workers of the ant Formica polyctena during dyadic aggression tests. Acta Neurobiol Exp. 2013;73:495-520.

121. Ceriani MF, Hogenesch JB, Yanovsky M, Panda S, Straume M, Kay SA. Genome-wide expression analysis in Drosophila reveals genes controlling circadian behavior. J Neurosci. 2002;22:9305-19.

122. Neckameyer WS, Cooper RL. GABA transporters in Drosophila melanogaster: molecular cloning, behavior, and physiology. Invertebr Neurosci. 1998;3:279-94.

123. George J, Blanford S, Domingue MJ, Thomas MB, Read AF, Baker TC. Reduction in host-finding behaviour in fungus-infected mosquitoes is correlated with reduction in olfactory receptor neuron responsiveness. Malaria J. 2011;10:219.

124. Poinar GO, Chabaud AG, Bain O. Rabbium aradoxus sp. n. (Seuratidae: Skrjabinelaziinae) maturing in Camponotus castaneus (Hymenoptera: Formicidae). P Helm Soc Wash. 1989;56:120-4.

125. Harmer SL, Panda S, Kay SA. Molecular bases of circadian rhythms. Ann Rev Cell Dev Biol. 2001;17:215-53.

126. Edgar RS, Green EW, Zhao Y, van Ooijen G, Olmedo M, Qin X, et al. Peroxiredoxins are conserved markers of circadian rhythms. Nature. 2012;485:459-64.

127. Ingram KK, Kutowoi A, Wurm Y, Shoemaker D, Meier R, Bloch G. The molecular clockwork of the fire ant Solenopsis invicta. PLoS One. 2012;7, e45715.

128. Dickmeis T, Foulkes NS. Glucocorticoids and circadian clock control of cell proliferation: at the interface between three dynamic systems. Mol Cell Endocrinol. 2011;331:11-22.

129. Masri S, Cervantes M, Sassone-Corsi P. The circadian clock and cell cycle: interconnected biological circuits. Curr Opin Cell Biol. 2013;25:730-4.

130. Yang X, Wood PA, Ansell CM, Quiton DF, Oh EY, Du-Quiton J, et al. The circadian clock gene Per1 suppresses cancer cell proliferation and tumor growth at specific times of day. Chronobiol Int. 2009;26:1323-39.

131. Zeng ZL, Wu MW, Sun J, Sun YL, Cai YC, Huang YJ, et al. Effects of the biological clock gene Bmal1 on tumour growth and anti-cancer drug activity. J Biochem. 2010;148:319-26.

132. Fu L, Pelicano H, Liu J, Huang P, Lee C. The circadian gene Period2 plays an important role in tumor suppression and DNA damage response in vivo. Cell. 2002;111:41-50.

133. Lin F, Chen Y, Li X, Zhao Q, Tan Z. Over-expression of circadian clock gene Bmal1 affects proliferation and the canonical Wnt pathway in $\mathrm{NIH}-3$ T3 cells. Cell Biochem Funct. 2013;31:166-72.

134. Gillies AR, Lieber RL. Structure and function of the skeletal muscle extracellular matrix. Muscle Nerve. 2011;44:318-31.

135. Clark KA, Bland JM, Beckerle MC. The Drosophila muscle LIM protein, Mlp84B, cooperates with D-titin to maintain muscle structural integrity. J Cell Sci. 2007;120:2066-77.

136. Smit AFAH R, Green P. RepeatMasker Open-3.0. 2010. p. 1996-2010. http://www.repeatmasker.org.

137. Jurka J, Kapitonov W, Pavlicek A, Klonowski P, Kohany O, Walichiewicz J. Repbase Update, a database of eukaryotic repetitive elements. Cytogenet Genome Res. 2005;110:462-7.
138. Price $A L$, Jones NC, Pevzner PA. De novo identification of repeat families in large genomes. Bioinformatics. 2005;21:i351-8.

139. Stanke M, Diekhans M, Baertsch R, Haussler D. Using native and syntenically mapped CDNA alignments to improve de novo gene finding. Bioinformatics. 2008;24:637-44.

140. Hu XZ YJ, Xiao GH, Zheng P, Xia YL, Zhang XY, St Leger RJ, et al. Genome survey uncovers the secrets of sex and lifestyle in caterpillar fungus. Chinese Sci Bull. 2013;58:2846-54.

141. Zheng P, Xia Y, Xiao G, Xiong C, Hu X, Zhang S, et al. Genome sequence of the insect pathogenic fungus Cordyceps militaris, a valued traditional Chinese medicine. Genome Biol. 2011;12:R116.

142. Gao Q, Jin K, Ying SH, Zhang Y, Xiao G, Shang Y, et al. Genome sequencing and comparative transcriptomics of the model entomopathogenic fungi Metarhizium anisopliae and M. acridum. PLoS Genet. 2011;7:e1001264.

143. Schardl CL, Young CA, Hesse U, Amyotte SG, Andreeva K, Calie PJ, et al. Plantsymbiotic fungi as chemical engineers: multi-genome analysis of the clavicipitaceae reveals dynamics of alkaloid loci. PLoS Genet. 2013;9, e1003323.

144. Ma LJ, van der Does HC, Borkovich KA, Coleman JJ, Daboussi MJ, Di Pietro A, et al. Comparative genomics reveals mobile pathogenicity chromosomes in Fusarium. Nature. 2010;464:367-73.

145. Cuomo CA, Guldener U, Xu JR, Trail F, Turgeon BG, Di Pietro A, et al. The Fusarium graminearum genome reveals a link between localized polymorphism and pathogen specialization. Science. 2007;317:1400-2.

146. Martinez D, Berka RM, Henrissat B, Saloheimo M, Arvas M, Baker SE, et al. Genome sequencing and analysis of the biomass-degrading fungus Trichoderma reesei (syn. Hypocrea jecorina). Nat Biotechnol. 2008;26:553-60.

147. Kubicek CP, Herrera-Estrella A, Seidl-Seiboth V, Martinez DA, Druzhinina IS, Thon $\mathrm{M}$, et al. Comparative genome sequence analysis underscores mycoparasitism as the ancestral life style of Trichoderma. Genome Biol. 2011;12:R40.

148. Dean RA, Talbot NJ, Ebbole DJ, Farman ML, Mitchell TK, Orbach MJ, et al. The genome sequence of the rice blast fungus Magnaporthe grisea. Nature. 2005;434:980-6.

149. Jones T, Federspiel NA, Chibana H, Dungan J, Kalman S, Magee BB, et al. The diploid genome sequence of Candida albicans. Proc Natl Acad Sci U S A. 2004;101:7329-34.

150. Nierman WC, Pain A, Anderson MJ, Wortman JR, Kim HS, Arroyo J, et al. Genomic sequence of the pathogenic and allergenic filamentous fungus Aspergillus fumigatus. Nature. 2005;438:1151-6.

151. Galagan JE, Calvo SE, Borkovich KA, Selker EU, Read ND, Jaffe D, et al. The genome sequence of the filamentous fungus Neurospora crassa. Nature. 2003:422:859-68.

152. Arnaud MB, Cerqueira GC, Inglis DO, Skrzypek MS, Binkley J, Chibucos MC, et al. The Aspergillus Genome Database (AspGD): recent developments in comprehensive multispecies curation, comparative genomics and community resources. Nucleic Acids Res. 2012;40:D653-9.

153. Ashburner M, Ball CA, Blake JA, Botstein D, Butler H, Cherry JM, et al. Gene ontology: tool for the unification of biology. The Gene Ontology Consortium. Nat Genet. 2000;25:25-9.

154. Hunter S, Apweiler R, Attwood TK, Bairoch A, Bateman A, Binns D, et al. InterPro: the integrative protein signature database. Nucleic Acids Res. 2009;37:D211-5.

155. Moriya Y, Itoh M, Okuda S, Yoshizawa AC, Kanehisa M. KAAS: an automatic genome annotation and pathway reconstruction server. Nucleic Acids Res. 2007;35:W182-5.

156. Rawlings ND, Waller M, Barrett AJ, Bateman A. MEROPS: the database of proteolytic enzymes, their substrates and inhibitors. Nucleic Acids Res. 2014:42:D503-9.

157. Petersen TN, Brunak S, von Heijne G, Nielsen H. SignalP 4.0: discriminating signal peptides from transmembrane regions. Nat Methods. 2011;8:785-6.

158. Krogh A, Larsson B, von Heijne G, Sonnhammer EL. Predicting transmembrane protein topology with a hidden Markov model: application to complete genomes. J Mol Biol. 2001;305:567-80.

159. Khaldi N, Seifuddin FT, Turner G, Haft D, Nierman WC, Wolfe KH, et al. SMURF: Genomic mapping of fungal secondary metabolite clusters. Fungal Genet Biol. 2010:47:736-41.

160. Li L, Stoeckert Jr CJ, Roos DS. OrthoMCL: identification of ortholog groups for eukaryotic genomes. Genome Res. 2003;13:2178-89.

161. Katoh K, Standley DM. MAFFT multiple sequence alignment software version 7: improvements in performance and usability. Mol Biol Evol. 2013;30:772-80. 
162. Castresana J. Selection of conserved blocks from multiple alignments for their use in phylogenetic analysis. Mol Biol Evol. 2000;17:540-52.

163. Stamatakis A. RAXML version 8: a tool for phylogenetic analysis and post-analysis of large phylogenies. Bioinformatics. 2014;30:1312-3.

164. Huson DH, Scornavacca C. Dendroscope 3: an interactive tool for rooted phylogenetic trees and networks. Syst Biol. 2012;61:1061-7.

165. Price MN, Dehal PS, Arkin AP. FastTree 2-approximately maximum-likelihood trees for large alignments. PLoS One. 2010;5, e9490.

166. de Bekker C, Bruning O, Jonker MJ, Breit TM, Wosten HA. Single cell transcriptomics of neighboring hyphae of Aspergillus niger. Genome Biol. 2011;12:R71.

167. Trapnell C, Pachter L, Salzberg SL. TopHat: discovering splice junctions with RNA-Seq. Bioinformatics. 2009:25:1105-11.

168. Langmead B, Trapnell C, Pop M, Salzberg SL. Ultrafast and memory-efficient alignment of short DNA sequences to the human genome. Genome Biol. 2009;10:R25.

169. Trapnell C, Williams BA, Pertea G, Mortazavi A, Kwan G, van Baren MJ, et al. Transcript assembly and quantification by RNA-Seq reveals unannotated transcripts and isoform switching during cell differentiation. Nat Biotechnol. 2010;28:511-5.

170. Roberts A, Trapnell C, Donaghey J, Rinn JL, Pachter L. Improving RNA-Seq expression estimates by correcting for fragment bias. Genome Biol. 2011;12:R22.

\section{Submit your next manuscript to BioMed Central and take full advantage of:}

- Convenient online submission

- Thorough peer review

- No space constraints or color figure charges

- Immediate publication on acceptance

- Inclusion in PubMed, CAS, Scopus and Google Scholar

- Research which is freely available for redistribution 